\title{
On surface-symmetric spacetimes with collisionless and charged matter
}

\author{
Sophonie Blaise Tchapnda
}

\begin{abstract}
Some future global properties of cosmological solutions for the EinsteinVlasov-Maxwell system with surface symmetry are presented. Global existence is proved, the homogeneous spacetimes are future complete, and the same is true for inhomogeneous plane-symmetric solutions with small initial data. In the latter case some decay properties are also obtained at late times. Similar but slightly weaker results hold for hyperbolic symmetry.
\end{abstract}

\section{Introduction and main results}

In general relativity the time evolution of self-gravitating collisionless particles can be modelled by the Einstein-Vlasov system. For surveys of results on that system see [1]. Cosmological spacetimes are those admitting a compact Cauchy hypersurface. In this case the particles are galaxies or even clusters of galaxies.

Results on expanding cosmological models with collisionless matter, a positive cosmological constant and surface symmetry have been obtained in [12, 13. In the present paper we want to examine what happens when the particles in the selfgravitating collisionless gas under consideration are charged. The Einstein-Vlasov system is then coupled to the Maxwell equations determining the electromagnetic field created by the charged particles. Considering this particular problem extends the knowledge on global dynamical properties of solutions of the Einstein equations. Adding the Maxwell equations could also help to answer the question why in the cosmology literature people usually talk about the magnetic field more than the electric field. If the accelerated expansion, which here is due to the positive cosmological constant, could let the latter decay faster than the former then this could be an explanation. This might also have some connection with the so-called Landau damping effect [15].

As known results related to the Einstein-Vlasov-Maxwell system we can mention a small data global existence theorem in the spherically symmetric asymptotically flat setting obtained in 5 . In the absence of Vlasov matter the asymptotic behaviour of solutions of that system with $T^{3}$-Gowdy symmetry was studied recently in [11. 
Let us now formulate our system. We suppose there are two species of charged particles, one of positive charge +1 and the other of negative charge -1 . All the particles are supposed to have the same rest mass equal to 1 , and to move forward in time so that the number densities $f^{+}$and $f^{-}$for positive and negative charge species respectively, are non-negative functions supported on the mass shell

$$
P M:=\left\{g_{\alpha \beta} p^{\alpha} p^{\beta}=-1, p^{0}>0\right\},
$$

a submanifold of the tangent bundle $T M$ of the space-time manifold $M$ with metric $g$ of signature -+++ . We use coordinates $\left(t, x^{a}\right)$ with zero shift and corresponding canonical momenta $p^{\alpha}$; Greek indices always run from 0 to 3 , and Latin ones from 1 to 3 . On the mass shell $P M$ the variable $p^{0}$ becomes a function of the remaining variables $\left(t, x^{a}, p^{b}\right)$ :

$$
p^{0}=\sqrt{-g^{00}} \sqrt{1+g_{a b} p^{a} p^{b}} .
$$

The Einstein-Vlasov-Maxwell system now reads

$$
\begin{gathered}
\partial_{t} f^{+}+\frac{p^{a}}{p^{0}} \partial_{x^{a}} f^{+}-\frac{1}{p^{0}}\left(\Gamma_{\beta \gamma}^{a} p^{\beta} p^{\gamma}+F_{\beta}{ }^{a} p^{\beta}\right) \partial_{p^{a}} f^{+}=0 \\
\partial_{t} f^{-}+\frac{p^{a}}{p^{0}} \partial_{x^{a}} f^{-}-\frac{1}{p^{0}}\left(\Gamma_{\beta \gamma}^{a} p^{\beta} p^{\gamma}-F_{\beta}{ }^{a} p^{\beta}\right) \partial_{p^{a}} f^{-}=0 \\
G_{\alpha \beta}+\Lambda g_{\alpha \beta}=8 \pi\left(T_{\alpha \beta}+\tau_{\alpha \beta}\right) \\
\nabla_{\alpha} F_{\beta \gamma}+\nabla_{\beta} F_{\gamma \alpha}+\nabla_{\gamma} F_{\alpha \beta}=0 \\
\nabla_{\alpha} F^{\alpha \beta}=J^{\beta} \\
T_{\alpha \beta}=-\int_{\mathbb{R}^{3}}\left(f^{+}+f^{-}\right) p_{\alpha} p_{\beta}|g|^{1 / 2} \frac{d p^{1} d p^{2} d p^{3}}{p_{0}} \\
\tau_{\alpha \beta}=F_{\alpha \gamma} F_{\beta}{ }^{\gamma}-\frac{g_{\alpha \beta}}{4} F^{\gamma \delta} F_{\gamma \delta} \\
J^{\beta}=\int_{\mathbb{R}^{3}}\left(f^{+}-f^{-}\right) p^{\beta}|g|^{1 / 2} \frac{d p^{1} d p^{2} d p^{3}}{p_{0}}
\end{gathered}
$$

where $p_{\alpha}=g_{\alpha \beta} p^{\beta}, \Gamma_{\beta \gamma}^{\alpha}$ are the Christoffel symbols, $|g|$ denotes the determinant of the metric $g, G_{\alpha \beta}$ the Einstein tensor, $\Lambda$ the cosmological constant, $F$ the electromagnetic field created by the charged particles, $J^{\beta}$ the total particle current density generated by the charged particles and $T_{\alpha \beta}$ and $\tau_{\alpha \beta}$ are the energy-momentum tensor for Vlasov and Maxwell matter respectively. 
A computation in normal coordinates shows that $\nabla_{\alpha} J^{\alpha}=0$. This equation is an expression of the conservation of charge. It can be shown as in [10] that $T_{\alpha \beta}$ satisfies the dominant energy condition i.e. $T_{\alpha \beta} V^{\alpha} W^{\beta} \geq 0$ for any two futurepointing timelike vectors $V^{\alpha}$ and $W^{\alpha}$. Let us show that the same is true for the Maxwell tensor $\tau_{\alpha \beta}$. Proving this is equivalent to show the weak-energy condition $\tau_{\alpha \beta} V^{\alpha} V^{\beta} \geq 0$ for all timelike vector $V^{\alpha}$, together with the property that $\tau_{\alpha \beta} V^{\beta}$ is non-spacelike for any future-pointing timelike vector $V^{\alpha}$. The proof of the latter can be deduced from the following identities which hold since $F$ is antisymmetric

$$
\tau_{\alpha \nu} \tau_{\beta}^{\nu}=\frac{1}{4}\left(\tau^{\gamma \delta} \tau_{\gamma \delta}\right) g_{\alpha \beta}, \quad \tau_{\alpha \beta} \tau^{\alpha \beta} \geq 0
$$

Contracting the first of these identities twice with $V^{\alpha}$ implies the following, using the second identity and the fact that $V^{\alpha}$ is timelike:

$$
\left(V^{\alpha} \tau_{\alpha \nu}\right)\left(\tau_{\beta}^{\nu} V^{\beta}\right)=\frac{1}{4}\left(\tau^{\gamma \delta} \tau_{\gamma \delta}\right) g_{\alpha \beta} V^{\alpha} V^{\beta} \leq 0
$$

and setting $P_{\nu}=V^{\alpha} \tau_{\alpha \nu}$, this means that $P_{\alpha} P^{\alpha} \leq 0$, that is $P_{\alpha}$ is non-spacelike as desired. Now proving the weak-energy condition is equivalent to show that $\tau_{00}$ is non-negative since we can choose an orthonormal frame such that $V^{\alpha}$ is the timelike vector of the frame. In such a frame $g_{00}=-1$ so that $\tau_{00}=\frac{1}{2} g^{a b} F_{0 a} F_{0 b}+$ $\frac{1}{4} F^{a b} F_{a b} \geq 0$ as the sum of spatial lengths of a vector and a tensor respectively.

In the present paper we adopt the definition of spacetimes with surface symmetry, i.e., spherical, plane or hyperbolic symmetry given in 8 . We write the system in areal coordinates, i.e. coordinates are chosen such that $R=t$, where $R$ is the area radius function on a surface of symmetry. The circumstances under which coordinates of this type exist are discussed in 2] for the Einstein-Vlasov system with vanishing $\Lambda$, and in 13 for the case with $\Lambda$. The analysis there can be extended to the situation under consideration here since the Maxwell tensor $\tau_{\alpha \beta}$ satisfies the dominant energy condition. In such coordinates the metric takes the form

$$
d s^{2}=-e^{2 \mu(t, r)} d t^{2}+e^{2 \lambda(t, r)} d r^{2}+t^{2}\left(d \theta^{2}+\sin _{k}^{2} \theta d \varphi^{2}\right)
$$

where

$$
\sin _{k} \theta:= \begin{cases}\sin \theta & \text { if } k=1 \\ 1 & \text { if } k=0 \\ \sinh \theta & \text { if } k=-1\end{cases}
$$

Here $t>0$, the functions $\lambda$ and $\mu$ are periodic in $r$ with period 1 . It can be shown as in [6] and [2] that due to the symmetry $f^{+}$and $f^{-}$can be written as a function of

$$
t, r, w:=e^{\lambda} p^{1} \text { and } L:=t^{4}\left(p^{2}\right)^{2}+t^{4} \sin _{k}^{2} \theta\left(p^{3}\right)^{2}, \text { with } r, w \in \mathbb{R} ; L \in[0,+\infty[.
$$

In these variables we have $p^{0}=e^{-\mu} \sqrt{1+w^{2}+L / t^{2}}$.

In surface symmetry the only non-zero components of $F$ are $F_{01}$ and $F_{23}$. Indeed setting $h:=g+e_{0} \otimes e_{0}-e_{1} \otimes e_{1}$, with $e_{0}=e^{-\mu} \frac{\partial}{\partial t}$ and $e_{1}=e^{-\lambda} \frac{\partial}{\partial r}$, the 
mapping $X^{\beta} \mapsto h_{\alpha}^{\beta} X^{\alpha}$ is the orthogonal projection on the tangent space of the orbit, and since the vector $Y_{\sigma}:=F_{\alpha \beta}\left(e_{0}\right)^{\alpha} h_{\sigma}^{\beta}$ is invariant under the symmetry group, it vanishes. This implies that $F_{02}=F_{03}=0$. Similarly, replacing $e_{0}$ by $e_{1}$ in the expression of $Y_{\sigma}$ yields $F_{12}=F_{13}=0$.

Now we can calculate the Maxwell equations in a coordinate frame. Equation (1.4) then implies the following, where $\left(\partial_{0}, \partial_{1}, \partial_{2}, \partial_{3}\right)=\left(\partial_{t}, \partial_{r}, \partial_{\theta}, \partial_{\varphi}\right)$ :

$$
\partial_{0} F_{23}=\partial_{1} F_{23}=\partial_{2} F_{01}=\partial_{3} F_{01}=0 \text {. }
$$

Using the fact that the mapping $\left(p^{1}, p^{2}, p^{3}\right) \mapsto\left(w, L, p^{3}\right)$ is one-to-one from $\left.\mathbb{R} \times\right] 0, \infty[\times \mathbb{R}$ to $\mathbb{R} \times] 0, \infty[\times \mathbb{R}$ and from $\mathbb{R} \times]-\infty, 0[\times \mathbb{R}$ to $\mathbb{R} \times] 0, \infty[\times \mathbb{R}$, one can compute the $J^{\alpha}$ 's and obtain $J^{2}=J^{3}=0$,

$$
J^{0}=-\frac{\pi}{t^{2}} e^{-\mu} \int_{-\infty}^{\infty} \int_{0}^{\infty}\left(f^{+}-f^{-}\right)(t, r, w, L) d L d w
$$

and

$$
J^{1}=-\frac{\pi}{t^{2}} e^{-\lambda} \int_{-\infty}^{\infty} \int_{0}^{\infty} \frac{w}{\sqrt{1+w^{2}+L / t^{2}}}\left(f^{+}-f^{-}\right)(t, r, w, L) d L d w .
$$

Equation (1.5) then implies

$$
\left\{\begin{array}{l}
\partial_{0}\left(\sqrt{|g|} F^{01}\right)=J^{1} \sqrt{|g|}, \quad \partial_{1}\left(\sqrt{|g|} F^{01}\right)=-J^{0} \sqrt{|g|} \\
\partial_{2}\left(\sqrt{|g|} F^{23}\right)=0, \quad \partial_{3}\left(\sqrt{|g|} F^{23}\right)=0 .
\end{array}\right.
$$

The non-zero components of the electric and magnetic parts of $F$ are $E^{1}=e^{\mu} F^{01}$ and $B_{1}=e^{-\mu} \sqrt{|g|} F^{23}$ respectively. Using these identities and recalling that $\sqrt{|g|}=t^{2} e^{\lambda+\mu} \sin _{k} \theta$, equations (1.10) and (1.11) lead to the following, where $c$ is an arbitrary constant and $E^{1}$ is denoted by $E$ :

$$
\begin{aligned}
\partial_{r}\left(t^{2} e^{\lambda} E(t, r)\right) & =\pi e^{\lambda} \int_{-\infty}^{\infty} \int_{0}^{\infty}\left(f^{+}-f^{-}\right)(t, r, w, L) d L d w \\
\partial_{t}\left(t^{2} e^{\lambda} E(t, r)\right) & =-e^{\mu} \pi \int_{-\infty}^{\infty} \int_{0}^{\infty} \frac{w}{\sqrt{1+w^{2}+L / t^{2}}}\left(f^{+}-f^{-}\right)(t, r, w, L) d L d w \\
B_{1}(t, r) & =c t^{-2} e^{\lambda(t, r)}
\end{aligned}
$$

After calculating the Vlasov equations in the variables $(t, r, w, L)$, the nontrivial components of the Einstein tensor, and the energy-momentum tensor and denoting by an upper dot or by prime the derivation with respect to $t$ or $r$ respectively, the complete Einstein-Vlasov-Maxwell system then reads as follows

$$
\partial_{t} f^{+}+\frac{e^{\mu-\lambda} w}{\sqrt{1+w^{2}+L / t^{2}}} \partial_{r} f^{+}-\left(\dot{\lambda} w+e^{\mu-\lambda} \mu^{\prime} \sqrt{1+w^{2}+L / t^{2}}-e^{\lambda+\mu} E\right) \partial_{w} f^{+}=0
$$




$$
\begin{gathered}
\partial_{t} f^{-}+\frac{e^{\mu-\lambda} w}{\sqrt{1+w^{2}+L / t^{2}}} \partial_{r} f^{-}-\left(\dot{\lambda} w+e^{\mu-\lambda} \mu^{\prime} \sqrt{1+w^{2}+L / t^{2}}+e^{\lambda+\mu} E\right) \partial_{w} f^{-}=0 \\
e^{-2 \mu}(2 t \dot{\lambda}+1)+k-\Lambda t^{2}=8 \pi t^{2} \rho \\
e^{-2 \mu}(2 t \dot{\mu}-1)-k+\Lambda t^{2}=8 \pi t^{2} p \\
\mu^{\prime}=-4 \pi t e^{\lambda+\mu} j \\
e^{-2 \lambda}\left(\mu^{\prime \prime}+\mu^{\prime}\left(\mu^{\prime}-\lambda^{\prime}\right)\right)-e^{-2 \mu}\left(\ddot{\lambda}+(\dot{\lambda}-\dot{\mu})\left(\dot{\lambda}+\frac{1}{t}\right)\right)+\Lambda=4 \pi q \\
\partial_{r}\left(t^{2} e^{\lambda} E\right)=t^{2} e^{\lambda} a \\
\partial_{t}\left(t^{2} e^{\lambda} E\right)=-t^{2} e^{\mu} b
\end{gathered}
$$

where

$$
\begin{aligned}
& \rho(t, r):=\frac{\pi}{t^{2}} \int_{-\infty}^{\infty} \int_{0}^{\infty} \sqrt{1+w^{2}+L / t^{2}}\left(f^{+}+f^{-}\right)(t, r, w, L) d L d w \\
& +\frac{1}{2}\left(e^{2 \lambda} E^{2}+c t^{-4}\right)=e^{-2 \mu}\left(T_{00}+\tau_{00}\right)(t, r), \\
& p(t, r):=\frac{\pi}{t^{2}} \int_{-\infty}^{\infty} \int_{0}^{\infty} \frac{w^{2}}{\sqrt{1+w^{2}+L / t^{2}}}\left(f^{+}+f^{-}\right)(t, r, w, L) d L d w \\
& -\frac{1}{2}\left(e^{2 \lambda} E^{2}+c t^{-4}\right)=e^{-2 \lambda}\left(T_{11}+\tau_{11}\right)(t, r), \\
& j(t, r):=\frac{\pi}{t^{2}} \int_{-\infty}^{\infty} \int_{0}^{\infty} w\left(f^{+}+f^{-}\right)(t, r, w, L) d L d w=-e^{\lambda+\mu} T_{01}(t, r), \\
& q(t, r):=\frac{\pi}{t^{4}} \int_{-\infty}^{\infty} \int_{0}^{\infty} \frac{L}{\sqrt{1+w^{2}+L / t^{2}}}\left(f^{+}+f^{-}\right)(t, r, w, L) d L d w \\
& +\left(e^{2 \lambda} E^{2}+c t^{-4}\right)=\frac{2}{t^{2}}\left(T_{22}+\tau_{22}\right)(t, r), \\
& a(t, r):=\frac{\pi}{t^{2}} \int_{-\infty}^{\infty} \int_{0}^{\infty}\left(f^{+}-f^{-}\right)(t, r, w, L) d L d w, \\
& b(t, r):=\frac{\pi}{t^{2}} \int_{-\infty}^{\infty} \int_{0}^{\infty} \frac{w}{\sqrt{1+w^{2}+L / t^{2}}}\left(f^{+}{ }_{-}^{-}\right)(t, r, w, L) d L d w .
\end{aligned}
$$


We prescribe initial data at some time $t=t_{0}>0$,

$$
\begin{array}{r}
f^{+}\left(t_{0}, r, w, L\right)=\stackrel{\circ}{f^{+}}(r, w, L), f^{-}\left(t_{0}, r, w, L\right)=\stackrel{\circ}{f^{-}}(r, w, L), \\
\lambda\left(t_{0}, r\right)=\stackrel{\circ}{\lambda}(r), \mu\left(t_{0}, r\right)=\stackrel{\circ}{\mu}(r), E\left(t_{0}, r\right)=\stackrel{\circ}{E}(r)
\end{array}
$$

and want to study the existence and behaviour of the corresponding solution for $t \in\left[t_{0},+\infty\right)$.

To this end we maintain the notation in 17, 12, 13, and follow their work wherever possible. The first step consists on generalizing the local existence result in [7] theorem 3.1] to the case of charge particles under study:

Theorem 1.1 Let $\stackrel{\circ}{f^{ \pm}} \in C^{1}\left(\mathbb{R}^{2} \times\left[0, \infty[)\right.\right.$ with $\stackrel{\circ}{f^{ \pm}}(r+1, w, L)=\stackrel{\circ}{f^{ \pm}}(r, w, L)$ for $(r, w, L) \in \mathbb{R}^{2} \times\left[0, \infty\left[, f^{ \pm} \geq 0\right.\right.$, and $w_{0}:=w_{0}^{+}+w_{0}^{-}, L_{0}:=L_{0}^{+}+L_{0}^{-}$with

$$
\begin{aligned}
& w_{0}^{ \pm}:=\sup \left\{|w| \mid(r, w, L) \in \operatorname{supp} f^{ \pm}\right\}<\infty \\
& L_{0}^{ \pm}:=\sup \left\{F \mid(r, w, L) \in \operatorname{supp} f^{ \pm}\right\}<\infty
\end{aligned}
$$

Let $\stackrel{\circ}{\lambda}, \stackrel{\circ}{E} \in C^{1}(\mathbb{R}), \stackrel{\circ}{\mu} \in C^{2}(\mathbb{R})$ with $\stackrel{\circ}{\lambda}(r)=\stackrel{\circ}{\lambda}(r+1), \stackrel{\circ}{\mu}(r)=\stackrel{\circ}{\mu}(r+1), \stackrel{\circ}{E}(r)=$ $\stackrel{\circ}{E}(r+1)$ for $r \in \mathbb{R}$, and

$$
\begin{aligned}
& \stackrel{\circ}{\mu}^{\prime}(r)=-4 \pi t_{0} e^{\stackrel{\circ}{\lambda}+\stackrel{\circ}{\mu}} j(r)=-\frac{4 \pi^{2}}{t_{0}} e^{\stackrel{\circ}{\lambda}+\stackrel{\circ}{\mu}} \int_{-\infty}^{\infty} \int_{0}^{\infty} w\left(\stackrel{\circ}{+}^{+}+\stackrel{\circ}{f^{-}}\right)(r, w, L) d L d w, \quad r \in \mathbb{R}, \\
& \partial_{r}\left(t_{0}^{2} e^{\stackrel{\circ}{\lambda}} \stackrel{\circ}{E}\right)=t_{0}^{2} e^{\stackrel{\circ}{\perp}} \stackrel{\circ}{a}=\pi e^{\stackrel{\circ}{\lambda}} \int_{-\infty}^{\infty} \int_{0}^{\infty}\left(\stackrel{\circ}{+}^{+}-\stackrel{\circ}{f^{-}}\right)(r, w, L) d L d w, \quad r \in \mathbb{R} .
\end{aligned}
$$

Then there exists a unique, right maximal, regular solution $\left(f^{+}, f^{-}, \lambda, \mu, E\right)$ of

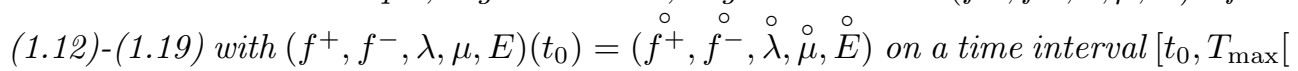
with $\left.\left.T_{\max } \in\right] t_{0}, \infty\right]$.

The regularity concept used in this statement is defined below.

The next theorem provides a continuation criterion used to prove that the solution exists on the whole time interval $\left[t_{0}, \infty[\right.$.

Theorem 1.2 Let $\left(f^{+}, f^{-}, \lambda, \mu, E\right)$ be a right maximal regular solution obtained in Theorem 1.1. If one has

$\sup \left\{|w| \mid(r, w, L) \in \operatorname{supp} f^{+}\right\}<\infty, \sup \left\{|w| \mid(r, w, L) \in \operatorname{supp} f^{-}\right\}<\infty$, $\sup \left\{\mu(t, r) \mid r \in \mathbb{R}, t \in\left[t_{0}, T_{\max }[\}<\infty, \sup \left\{\left(e^{\lambda} E\right)(t, r) \mid r \in \mathbb{R}, t \in\left[t_{0}, T_{\max }[\}<\infty\right.\right.\right.\right.$ then $T_{\max }=\infty$. 
In the following we claim that among the conditions given in the previous theorem there is one which implies the others.

Proposition 1.3 The condition

$$
\sup \left\{\mu(t, r) \mid r \in \mathbb{R}, t \in\left[t_{0}, T_{\max }[\}<\infty\right.\right.
$$

is sufficient in order to conclude that $T_{\max }=\infty$.

We can then state

Theorem 1.4 For initial data as in Theorem 1.1 with $t_{0}^{2}>1 / \Lambda$ in the case of spherical symmetry, the solution of the surface-symmetric Einstein-Vlasov-Maxwell system with positive cosmological constant, written in areal coordinates, exist for all $t \in\left[t_{0}, \infty[\right.$ where $t$ denotes the area radius of the surfaces of symmetry of the induced spacetime.

Once the existence of solutions is proven, one would like to study their asymptotic behaviour at late times. In particular an important point is to know whether the spacetime obtained is future complete or not. This seems not to be easily achieved by a direct argument for the generic data case of the inhomogeneous Einstein-Vlasov-Maxwell system. Nevertheless it works out in the spatially homogeneous case, as well as in the inhomogeneous plane-symmetric case under an additional assumption on the initial data.

Let us examine spatially homogeneous solutions. These correspond to LRS (locally rotationally symmetric) models of Bianchi type I and type III and KantowskiSachs type for plane, hyperbolic and spherical symmetry respectively. We refer to 9] for a detailed discussion on these models. We use the same notation as in [4, where the case of uncharged particles has been studied. Most of the results obtained in that case apply also if the particles are charged, the exception being those for which the proof involves matter terms. We will also use results from [14 in which the only requirement for the energy-momentm tensor is to satisfy the dominant and strong energy conditions, these are valid in the case under investigation. Let us formulate our system in Bianchi symmetry.

The spacetime is considered as a manifold $G \times I, I$ being an open interval and $G$ a simply connected three-dimensional Lie group. The metric has the form

$$
d s^{2}=-d \tau^{2}+g_{i j} e^{i} \otimes e^{j},
$$

where $\left\{e_{i}\right\}$ is a left invariant frame and $\left\{e^{i}\right\}$ the dual coframe.

The Einstein constraint equations are

$$
\begin{array}{r}
R-k_{i j} k^{i j}+\left(k_{i j} g^{i j}\right)^{2}=16 \pi\left(T_{00}+\tau_{00}\right)+2 \Lambda \\
\nabla^{i} k_{i j}=-8 \pi T_{0 j} .
\end{array}
$$


The evolution equations are

$$
\begin{aligned}
& \partial_{t} g_{i j}=-2 k_{i j} \\
& \begin{aligned}
\partial_{t} k_{i j}=R_{i j}+ & \left(k_{l m} g^{l m}\right) k_{i j}-2 k_{i l} k_{j}^{l}-8 \pi\left(T_{i j}+\tau_{i j}\right) \\
& \quad-4 \pi\left(T_{00}+\tau_{00}\right)+4 \pi\left(T_{l m}+\tau_{l m}\right) g^{l m} g_{i j}-\Lambda g_{i j},
\end{aligned}
\end{aligned}
$$

where

$$
\begin{gathered}
T_{00}+\tau_{00}=\int\left(f^{+}+f^{-}\right)(\tau, v)\left(1+g_{r s} v^{r} v^{s}\right)^{1 / 2}(\operatorname{det} g)^{1 / 2} d v \\
+F_{0 \gamma} F_{0}{ }^{\gamma}+\frac{1}{4} F_{\gamma \delta} F^{\gamma \delta} \\
T_{0 i}=\int\left(f^{+}+f^{-}\right)(\tau, v) v_{i}(\operatorname{det} g)^{1 / 2} d v \\
T_{i j}+\tau_{i j}=\int\left(f^{+}+f^{-}\right)(\tau, v) v_{i} v_{j}\left(1+g_{r s} v^{r} v^{s}\right)^{-1 / 2}(\operatorname{det} g)^{1 / 2} d v \\
+F_{i \gamma} F_{j} \gamma-\frac{g_{i j}}{4} F_{\gamma \delta} F^{\gamma \delta},
\end{gathered}
$$

with $v:=\left(v^{1}, v^{2}, v^{3}\right)$ and $d v:=d v^{1} d v^{2} d v^{3}$.

The Vlasov equations are

$$
\begin{gathered}
\partial_{\tau} f^{+}+\left[2 k_{j}^{i} v^{j}-\left(1+g_{r s} v^{r} v^{s}\right)^{-1 / 2} \gamma_{m n}^{i} v^{m} v^{n}-\left(F_{0}{ }^{i}+F_{j} \frac{i}{v^{0}}\right)\right] \partial_{v^{i}} f^{+}=0 \\
\partial_{\tau} f^{-}+\left[2 k_{j}^{i} v^{j}-\left(1+g_{r s} v^{r} v^{s}\right)^{-1 / 2} \gamma_{m n}^{i} v^{m} v^{n}+\left(F_{0}{ }^{i}+F_{j} i \frac{v^{j}}{v^{0}}\right)\right] \partial_{v^{i}} f^{-}=0
\end{gathered}
$$

where $\gamma_{m n}^{i}=\frac{1}{2} g^{i k}\left(-C_{n k}^{l} g_{m l}+C_{k m}^{l} g_{n l}+C_{m n}^{l} g_{k l}\right), C_{j k}^{i}$ are the structure constants of the Lie algebra of $G$.

Note that in LRS Bianchi symmetry the only non-zero components of $F$ are $F^{01}$ and $F^{23}$. The Maxwell equations (1.4) allow us to obtain an explicit expression for the magnetic part $F^{23}$ of $F$. Thus the remaining unknown for the Maxwell equations is the electric part $F^{01}$ of $F$. For a Bianchi model the Maxwell equations (1.5) take the following form

$$
\partial_{\tau}\left[(\operatorname{det} g)^{1 / 2} F^{0 \beta}\right]+C_{i j}^{i} F^{j \beta}(\operatorname{det} g)^{1 / 2}=J^{\beta}(\operatorname{det} g)^{1 / 2}
$$

which yields for $\beta=0$ and $\beta=i$ respectively

$$
\begin{aligned}
C_{i j}^{i} F^{j 0} & =J^{0} \\
\partial_{\tau} F^{0 i}-(t r k) F^{0 i}+C_{k j}^{k} F^{j i} & =J^{i},
\end{aligned}
$$


here

$$
\begin{aligned}
J^{0} & =\int\left(f^{+}-f^{-}\right)(\tau, v)(\operatorname{det} g)^{1 / 2} d v \\
J^{i} & =\int\left(f^{+}-f^{-}\right)(\tau, v) v^{i}\left(1+g_{r s} v^{r} v^{s}\right)^{-1 / 2}(\operatorname{det} g)^{1 / 2} d v .
\end{aligned}
$$

Setting $k_{i j}=\frac{1}{3}\left(k_{l m} g^{l m}\right) g_{i j}+\sigma_{i j}, \sigma_{i j}$ being the trace free part of the second fundamental form $k_{i j}$, and using the Hamiltonian constraint (1.26) we obtain

$$
\frac{1}{3}\left(k_{i j} g^{i j}\right)^{2}=\frac{-R}{2}+\frac{1}{2} \sigma_{i j} \sigma^{i j}+8 \pi \rho+\Lambda,
$$

then using the fact that $R \leq 0$ (cf. [14]), it follows by the Proposition 1 in [4] that the matter energy density is bounded by

$$
\rho \leq C e^{-2 \gamma \tau} .
$$

We can prove the following

Theorem 1.5 Let $f^{ \pm}(0, v)$ be a nonnegative $C^{1}$ function with compact support. Let $\left(g_{i j}(0), k_{i j}(0), f^{+}(0, v), f^{-}(0, v), F^{0 i}(0)\right)$ be an initial data set for the evolution equations (1.28), (1.29), the Vlasov equations (1.33), (1.34) and the Maxwell equation (1.36), which has Bianchi symmetry and satisfies the constraint equations (1.26), (1.27), and (1.35). Then the corresponding solution of the Einstein-VlasovMaxwell system is a future complete spacetime for causal trajectories.

In the inhomogeneous case the result in the latter theorem can be proved in plane symmetry for small initial data. We have the following

Theorem 1.6 Consider any solution of Einstein-Vlasov-Maxwell system with positive cosmological constant in surface symmetry written in areal coordinates, with initial data as in theorem 1.4 Let $\delta$ be a positive constant and suppose the following inequalities hold:

$$
\begin{gathered}
\left|t_{0} \dot{\lambda}\left(t_{0}\right)-1\right| \leq \delta,\left|\left(e^{-\lambda} \mu^{\prime}\right)\left(t_{0}\right)\right| \leq \delta,\left|\left(e^{\lambda} E\right)\left(t_{0}\right)\right| \leq \delta \\
\left|\Lambda t_{0}^{2} e^{2 \mu\left(t_{0}\right)}-3-3 k e^{2 \mu\left(t_{0}\right)}\right| \leq \delta, \bar{w}\left(t_{0}\right) \leq \delta, c \leq \delta,
\end{gathered}
$$

where $\bar{w}(t)$ denotes the maximum of $w$ over the support of $f^{+}(t)$ or $f^{-}(t)$. Then if $\delta$ is sufficiently small, the following properties hold at late times:

$$
\begin{gathered}
t \dot{\lambda}-1=O\left(t^{-2}\right), e^{-\lambda} \mu^{\prime}=O\left(t^{-2}\right), e^{\lambda} E=O\left(t^{-2}\right), \\
\Lambda t^{2} e^{2 \mu}-3-3 k e^{2 \mu}=O\left(t^{-3}\right), \bar{w}=O\left(t^{-1}\right) .
\end{gathered}
$$

Furthermore the spacetime is future complete for causal trajectories.

The rest of the paper is organized as follows. In section 2 we present some preliminary results that we use to prove Theorem 1.1 in section 3 . The proof for the other results is also given in section 3 . 


\section{Preliminaries}

The regularity properties required for a solution are as in [7].

Definition 2.1 Let $I \subset] 0, \infty[$ be an interval

(a) $f^{ \pm} \in C^{1}\left(I \times \mathbb{R}^{2} \times\left[0, \infty[)\right.\right.$ is regular, if $f^{ \pm}(t, r+1, w, L)=f^{ \pm}(t, r, w, L)$ for $(t, r, w, L) \in I \times \mathbb{R}^{2} \times\left[0, \infty\left[, f^{ \pm} \geq 0\right.\right.$, and $\operatorname{supp} f^{ \pm}(t, r, . .$.$) is compact, uniformly$ in $r$ and locally uniformly in $t$.

(b) $\rho$ (or $p, j, q, a, b) \in C^{1}(I \times \mathbb{R})$ is regular, if $\rho(t, r+1)=\rho(t, r)$ for $(t, r) \in I \times \mathbb{R}$

(c) $\lambda \in C^{1}(I \times \mathbb{R})$ is regular, if $\dot{\lambda} \in C^{1}(I \times \mathbb{R})$ and $\lambda(t, r+1)=\lambda(t, r)$ for $(t, r) \in I \times \mathbb{R}$

(d) $\mu \in C^{1}(I \times \mathbb{R})$ is regular, if $\mu^{\prime} \in C^{1}(I \times \mathbb{R})$ and $\mu(t, r+1)=\mu(t, r)$ for $(t, r) \in I \times \mathbb{R}$.

(e) $E($ or $\tilde{\mu}) \in C^{1}(I \times \mathbb{R})$ is regular, if $E(t, r+1)=E(t, r)$ for $(t, r) \in I \times \mathbb{R}$.

It is possible to solve each equation of the system (1.12)-(1.15), (1.19), when the other unknowns are given. This is the content of the following

Proposition 2.2 Let $\overline{f^{+}}, \overline{f^{-}}, \bar{\lambda}, \bar{\mu}, \bar{E}$ be regular for $\left.(t, r) \in I \times \mathbb{R}, I \subset\right] 0, \infty[$ an interval with $t_{0} \in I$. Replace $f^{+}, f^{-}, \lambda, \mu, E$ respectively by $\overline{f^{+}}, \overline{f^{-}}, \bar{\lambda}, \bar{\mu}, \bar{E}$ in $\rho$, $b, p$ to define $\bar{\rho}, \bar{b}$ and $\bar{p}$. Suppose that $f^{+}, \stackrel{\circ}{f^{-}} \in C^{1}\left(\mathbb{R}^{2} \times\left[0, \infty[), \stackrel{\circ}{\lambda}, \stackrel{\circ}{\mu}, \stackrel{\circ}{E} \in C^{1}(\mathbb{R})\right.\right.$ and are periodic of period 1 in $r$. Assume that

$$
\frac{t_{0}\left(e^{-2 \stackrel{\circ}{\mu}(r)}+k\right)}{t}-k+\frac{8 \pi}{t} \int_{t}^{t_{0}} s^{2} \bar{p}(s, r) d s+\frac{\Lambda}{3 t}\left(t^{3}-t_{0}^{3}\right)>0,(t, r) \in I \times \mathbb{R} .
$$

Then the system

$\partial_{t} f^{+}+\frac{e^{\bar{\mu}-\bar{\lambda}} w}{\sqrt{1+w^{2}+L / t^{2}}} \partial_{r} f^{+}-\left(\dot{\bar{\lambda}} w+e^{\bar{\mu}-\bar{\lambda}} \bar{\mu}^{\prime} \sqrt{1+w^{2}+L / t^{2}}-e^{\bar{\lambda}+\bar{\mu}} \bar{E}\right) \partial_{w} f^{+}=0$

$\partial_{t} f^{-}+\frac{e^{\bar{\mu}-\bar{\lambda}} w}{\sqrt{1+w^{2}+L / t^{2}}} \partial_{r} f^{-}-\left(\dot{\bar{\lambda}} w+e^{\bar{\mu}-\bar{\lambda}} \bar{\mu}^{\prime} \sqrt{1+w^{2}+L / t^{2}}+e^{\bar{\lambda}+\bar{\mu}} \bar{E}\right) \partial_{w} f^{-}=0$

$$
\begin{gathered}
e^{-2 \mu}(2 t \dot{\lambda}+1)+k-\Lambda t^{2}=8 \pi t^{2} \bar{\rho} \\
e^{-2 \mu}(2 t \dot{\mu}-1)-k+\Lambda t^{2}=8 \pi t^{2} \bar{p} \\
\partial_{t}\left(t^{2} e^{\lambda} E\right)=-t^{2} e^{\mu} \bar{b}
\end{gathered}
$$


has a unique, regular solution $\left(f^{+}, f^{-}, \lambda, \mu, E\right)$ on $I \times \mathbb{R}$ with $f^{+}\left(t_{0}\right)=f^{+}$, $f^{-}\left(t_{0}\right)=\stackrel{\circ}{f^{-}}, \lambda\left(t_{0}\right)=\stackrel{\circ}{\lambda}, \mu\left(t_{0}\right)=\stackrel{\circ}{\mu}$ and $E\left(t_{0}\right)=\stackrel{\circ}{E}$. The solution is given by

$$
\begin{aligned}
& f^{ \pm}(t, r, w, L)=\stackrel{\circ}{f^{ \pm}}\left(\left(R^{ \pm}, W^{ \pm}\right)\left(t_{0}, t, w, L\right), L\right) \\
& e^{-2 \mu(t, r)}=\frac{t_{0}\left(e^{-2 \stackrel{\circ}{\mu}(r)}+k\right)}{t}-k+\frac{8 \pi}{t} \int_{t}^{t_{0}} s^{2} \bar{p}(s, r) d s+\frac{\Lambda}{3 t}\left(t^{3}-t_{0}^{3}\right) \\
& \dot{\lambda}(t, r)=4 \pi t e^{2 \mu(t, r)} \bar{\rho}(t, r)-\frac{1+k e^{2 \mu(t, r)}}{2 t}+\frac{\Lambda}{2} t e^{2 \mu(t, r)} \\
& \lambda(t, r)=\stackrel{\circ}{\lambda}(r)-\int_{t}^{t_{0}} \dot{\lambda}(s, r) d s \\
& E(t, r)=t^{-2} e^{-\lambda(t, r)}\left(t_{0}^{2} e^{\stackrel{\circ}{\lambda}(r)} \stackrel{\circ}{E}(r)-\int_{t_{0}}^{t} s^{2} e^{\mu(s, r)} \bar{b}(s, r) d s\right)
\end{aligned}
$$

where $\left(R^{ \pm}, W^{ \pm}\right)$is the solution of the characteristic system

$$
\frac{d}{d s}(r, w)=\left(\frac{e^{\bar{\mu}-\bar{\lambda}} w}{\sqrt{1+w^{2}+L / t^{2}}},-\dot{\bar{\lambda}} w-e^{\bar{\mu}-\bar{\lambda}} \bar{\mu}^{\prime} \sqrt{1+w^{2}+L / t^{2}} \pm e^{\bar{\lambda}+\bar{\mu}} \bar{E}\right)
$$

satisfying $\left(R^{ \pm}, W^{ \pm}\right)(t, t, r, w, L)=(r, w)$. If $I=\left[t_{0}, T[\right.$ with $\left.T \in] t_{0}, \infty\right]$ then there exists some $\left.\left.T^{\star} \in\right] t_{0}, T\right]$ such that condition (2.1) holds on $\left[t_{0}, T^{\star}\left[\times \mathbb{R} . T^{\star}\right.\right.$ depends on $\bar{p}$.

Proof Integrating (2.6) with respect to $t$ over $\left[t_{0}, t\right]$ gives (2.11). The rest of the proof is similar to those of propositions 2.2 and $2.3,1$ ) in $[12$.

In order to solve the system (1.12)-(1.19), it will be enough to concentrate on the subsystem (1.12)-(1.15), (1.19), as can be seen in

Proposition 2.3 The subsystem (1.12)-(1.15), (1.19) is equivalent to the full system (1.12)-(1.19), provided the initial data satisfy (1.16) and (1.18) at $t=t_{0}$.

Proof Under the assumption that the subsystem (1.12)-(1.15), (1.19) is satisfied as well as equations (1.16) and (1.18) for $t=t_{0}$, we should prove that (1.16)-(1.18) hold for all $t$. Integrating (1.19) over $\left[t_{0}, t\right]$ with respect to $t$ and differentiating the resulting equation with respect to $r$ yields

$$
\partial_{r}\left(t^{2} e^{\lambda} E\right)=t_{0}^{2} e^{\stackrel{\circ}{a} a}+\int_{t}^{t_{0}} s^{2} e^{\mu}\left(b^{\prime}+\mu^{\prime} b\right) d s
$$

Using equations (1.12) and (1.13) and integration by parts with respect to $t$ and $w$ leads to an expression for $\int_{t}^{t_{0}} s^{2} e^{\mu} b^{\prime} d s$ so that (2.13) implies

$$
\partial_{r}\left(t^{2} e^{\lambda} E\right)=t^{2} e^{\lambda} a+\pi \int_{t}^{t_{0}} \int_{-\infty}^{\infty} \int_{0}^{\infty} e^{2 \lambda+\mu} E\left(f^{+}+f^{-}\right)(s, r, w, L) d L d w d s .
$$


Computing the conservation law $\nabla_{\alpha} J^{\alpha}=0$ in coordinates $(t, r, w, L)$ and integrating the resulting equation with respect to $t$ yields

$$
\pi \int_{t}^{t_{0}} \int_{-\infty}^{\infty} \int_{0}^{\infty} e^{2 \lambda+\mu} E\left(f^{+}+f^{-}\right)(s, r, w, L) d L d w d s=0
$$

and so (2.14) becomes $\partial_{r}\left(t^{2} e^{\lambda} E\right)=t^{2} e^{\lambda} a$ that is (1.18) holds for all $t$. Using the latter and the same argument of the proof in [7. Prop. 2.2] we can show that (1.16) and (1.17) hold for all $t$ as well.

The latter proposition shows that equations (1.16) and (1.18) are invariant under evolution. So they will be considered as constraint equations on initial data (at $t=t_{0}$ ). They can be solved :

Proposition 2.4 The constraint equations $\stackrel{\circ}{\mu}^{\prime}(r)=-4 \pi t_{0} e^{\stackrel{\circ}{\lambda}+\stackrel{\circ}{\mu}} j(r)$ and

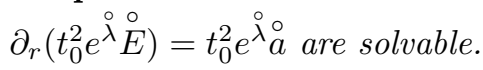

Proof To solve these equations we need to impose the following conditions, because $\left(e^{\stackrel{\circ}{\perp}} \stackrel{)}{)}(r)\right.$ and $e^{-\stackrel{\circ}{\mu}(r)}$ are periodic in $r$ with period 1 :

$$
\int_{0}^{1} \int_{-\infty}^{\infty} \int_{0}^{\infty} e^{\stackrel{\circ}{\lambda}}\left(f^{+}-\stackrel{\circ}{f^{-}}\right)(r, w, L) d L d w d r=0
$$

and

$$
I\left(\stackrel{\circ}{f^{ \pm}}\right):=\frac{4 \pi^{2}}{t_{0}} \int_{0}^{1} \int_{-\infty}^{\infty} \int_{0}^{\infty} e^{\stackrel{\circ}{\lambda}} w \stackrel{\circ}{ \pm}(r, w, L) d L d w d r=0
$$

Choosing $\stackrel{\circ}{\lambda}$ freely, and $\overline{f^{+}}, \overline{f^{-}}$non negative functions, the argument of the proof in [12, Remark 2.4] applies.

Remark Note that considering a model with more than one species of particles is important in order to prove the solvability of the second constraint equation above. Indeed if we had only one species of particles the integral $\int_{0}^{1} \int_{-\infty}^{\infty} \int_{0}^{\infty} e^{\stackrel{\circ}{\lambda}} f(r, w, L) d L d w d r$ would never vanish, except if $f$ is identically zero.

\section{Proofs}

\subsection{Proof of Theorem 1.1}

Instead of considering the subsystem (1.12)-(1.15), (1.19), an idea used in [7, that we follow here, is to consider an auxiliary system consisting of

$\partial_{t} f^{+}+\frac{e^{\mu-\lambda} w}{\sqrt{1+w^{2}+L / t^{2}}} \partial_{r} f^{+}-\left(\dot{\lambda} w+e^{\mu-\lambda} \tilde{\mu} \sqrt{1+w^{2}+L / t^{2}}-e^{\lambda+\mu} E\right) \partial_{w} f^{+}=0$, 
$\partial_{t} f^{-}+\frac{e^{\mu-\lambda} w}{\sqrt{1+w^{2}+L / t^{2}}} \partial_{r} f^{-}-\left(\dot{\lambda} w+e^{\mu-\lambda} \tilde{\mu} \sqrt{1+w^{2}+L / t^{2}}+e^{\lambda+\mu} E\right) \partial_{w} f^{-}=0$,

together with (1.14), (1.15), 1.19) and

$$
\tilde{\mu}=-4 \pi t e^{\lambda+\mu} j .
$$

Next by proving that $\mu^{\prime}=\tilde{\mu}$ it is easy to show that if $\left(f^{+}, f^{-}, \lambda, \mu, \tilde{\mu}, E\right)$ is a regular solution of (3.1), (3.2), (1.14), (1.15), (3.3), (1.19) on some time interval $I \subset] 0, \infty\left[\right.$ with $t_{0} \in I$, and with initial data satisfying (1.16) and (1.18) for $t=t_{0}$, then $\left(f^{+}, f^{-}, \lambda, \mu, E\right)$ solves (1.12)-(1.19).

As in [7, the solution of the auxiliary system above is used to construct a sequence of iterative solutions.

Let $\stackrel{\circ}{\tilde{\mu}}:=\stackrel{\circ}{\mu}, \lambda_{0}(t, r):=\stackrel{\circ}{\lambda}(r), \mu_{0}(t, r):=\stackrel{\circ}{\mu}(r), \tilde{\mu}_{0}(t, r):=\stackrel{\circ}{\tilde{\mu}}(r), E_{0}(t, r):=$ $\stackrel{\circ}{E}(r)$ for $\left.t \in] 0, t_{0}\right], r \in \mathbb{R} ; T_{0}=\infty$. If $\lambda_{n-1}, \mu_{n-1}, \tilde{\mu}_{n-1}, E_{n-1}$ are already defined and regular on $\left[t_{0}, T_{n-1}\left[\times \mathbb{R}\right.\right.$ with $T_{n-1} \geq 0$ then let

$$
\begin{aligned}
G_{n-1}^{ \pm}(t, r, w, L) & :=\left(\frac{w e^{\mu_{n-1}-\lambda_{n-1}}}{\sqrt{1+w^{2}+L / t^{2}}},-\dot{\lambda}_{n-1} w\right. \\
& \left.-e^{\mu_{n-1}-\lambda_{n-1}} \tilde{\mu}_{n-1} \sqrt{1+w^{2}+L / t^{2}} \pm e^{\lambda_{n-1}+\mu_{n-1}} E_{n-1}\right)
\end{aligned}
$$

for $t \in\left[t_{0}, T_{n-1}\left[\right.\right.$ and denote by $\left(R_{n}^{ \pm}, W_{n}^{ \pm}\right)(s, t, r, w, L)$ the solution of the characteristic system

$$
\frac{d}{d s}\left(R^{ \pm}, W^{ \pm}\right)=G_{n-1}^{ \pm}(s, R, W, L)
$$

with initial data

$$
\left(R_{n}^{ \pm}, W_{n}^{ \pm}\right)(t, t, r, w, L)=(r, w), \quad(t, r, w, L) \in\left[t_{0}, T_{n-1}\left[\times \mathbb{R}^{2} \times[0, \infty[.\right.\right.
$$

Define

$$
f_{n}^{ \pm}(t, r, w, L):=\stackrel{\circ}{f^{ \pm}}\left(\left(R_{n}^{ \pm}, W_{n}^{ \pm}\right)\left(t_{0}, t, r, w, L\right), L\right),
$$

that is, $f_{n}^{ \pm}$is the solution of

$$
\begin{aligned}
\partial_{t} f_{n}^{ \pm} & +\frac{w e^{\mu_{n-1}-\lambda_{n-1}}}{\sqrt{1+w^{2}+L / t^{2}}} \partial_{r} f_{n}^{ \pm}-\left(\dot{\lambda}_{n-1} w+\right. \\
& \left.e^{\mu_{n-1}-\lambda_{n-1}} \tilde{\mu}_{n-1} \sqrt{1+w^{2}+L / t^{2}} \mp e^{\lambda_{n-1}+\mu_{n-1}} E_{n-1}\right) \partial_{w} f_{n}^{ \pm}=0
\end{aligned}
$$


with $f_{n}^{ \pm}\left(t_{0}\right)=f^{ \pm}$, and define $\rho_{n}, p_{n}, j_{n}, q_{n}, a_{n}, b_{n}$ by the integrals (1.20)-(1.25) with $f^{ \pm}, E, \lambda$ replaced by $f_{n}^{ \pm}, E_{n-1}, \lambda_{n-1}$ respectively. Define

$$
\begin{array}{r}
T_{n}:=\sup \left\{t^{\prime} \in\right] t_{0}, T_{n-1}\left[\mid \frac{t_{0}\left(e^{-2 \stackrel{\circ}{\mu}(r)}+k\right)}{t}-k-\frac{8 \pi}{t} \int_{t_{0}}^{t} s^{2} p_{n}(s, r) d s\right. \\
\left.+\frac{\Lambda}{3 t}\left(t^{3}-t_{0}^{3}\right)>0, r \in \mathbb{R}, t \in\left[t_{0}, t^{\prime}\right]\right\} .
\end{array}
$$

Using Proposition 2.2 let

$$
\begin{gathered}
e^{-2 \mu_{n}(t, r)}:=\frac{t_{0}\left(e^{-2 \mu \stackrel{\circ}{ }(r)}+k\right)}{t}-k+\frac{8 \pi}{t} \int_{t}^{t_{0}} s^{2} p_{n}(s, r) d s+\frac{\Lambda}{3 t}\left(t^{3}-t_{0}^{3}\right), \\
\dot{\lambda}_{n}(t, r):=4 \pi t e^{2 \mu_{n}(t, r)} \rho_{n}(t, r)-\frac{1+k e^{2 \mu_{n}(t, r)}}{2 t}+\frac{\Lambda}{2} t e^{2 \mu_{n}(t, r)}, \\
\lambda_{n}(t, r):=\stackrel{\circ}{\lambda}(r)-\int_{t}^{t_{0}} \dot{\lambda}_{n}(s, r) d s, \\
\tilde{\mu}_{n}(t, r):=-4 \pi t e^{\lambda_{n}+\mu_{n}} j_{n}(t, r), \\
E_{n}(t, r):=t^{-2} e^{-\lambda_{n}(t, r)}\left(t_{0}^{2} e^{\stackrel{\circ}{\lambda}(r)} \stackrel{\circ}{E}(r)-\int_{t_{0}}^{t} s^{2} e^{\mu_{n}(s, r)} b_{n}(s, r) d s\right) .
\end{gathered}
$$

Subtracting equation (3.5) corresponding to $f^{+}$by the one corresponding to $f^{-}$, integrating the resulting equation with respect to $w$ and $L$ and integrating by parts with respect to $w$ yield

$$
\partial_{r}\left(t^{2} e^{\mu_{n}} b_{n}\right)=\left(\dot{\lambda}_{n}-\dot{\lambda}_{n-1}\right) e^{\lambda_{n-1}-\mu_{n-1}+\mu_{n}} t^{2} a_{n}-e^{\lambda_{n-1}-\lambda_{n}-\mu_{n-1}+\mu_{n}} \partial_{t}\left(t^{2} e^{\lambda_{n}} a_{n}\right),
$$

so that multiplying (3.10) by $t^{2} e^{\lambda_{n}}$ and differentiating the resulting equation with respect to $r$ lead to

$$
\begin{aligned}
\partial_{r}\left(t^{2} e^{\lambda_{n}} E_{n}\right)= & t^{2} a_{n} e^{\lambda_{n-1}-\mu_{n-1}}-\int_{t_{0}}^{t} s^{2}\left(\mu_{n}^{\prime}-\tilde{\mu}_{n-1}\right) e^{\mu_{n}} b_{n} d s \\
& +\int_{t_{0}}^{t} s^{2}\left(\dot{\mu}_{n}-\dot{\mu}_{n-1}\right) a_{n} e^{\mu_{n}-\mu_{n-1}+\lambda_{n-1}} d s .
\end{aligned}
$$

We split the proof of theorem 1.1 into several lemmas. From now on $\|\cdot\|$ denotes the $L^{\infty}$-norm on the function space in question, the numerical constant $C$ may change from line to line and does not depend on $n$ or $t$ or the initial data. Firstly we prove:

Lemma 3.1 The sequences $\mu_{n}, \lambda_{n}, \dot{\lambda}_{n}, \rho_{n}, p_{n}, E_{n}, j_{n}, a_{n}, b_{n}, \tilde{\mu}_{n} e^{\mu_{n}-\lambda_{n}}$ are uniformly bounded in $n$. 
Proof Define $P_{n}(t):=\left(P_{n}^{+}+P_{n}^{-}\right)(t)$ with

$$
\begin{aligned}
P_{n}^{ \pm}(t) & :=\sup \left\{|w| \mid(r, w, L) \in \operatorname{supp} f_{n}^{ \pm}(t)\right\}, \quad t \in\left[t_{0}, T_{n}[, \quad \text { and }\right. \\
Q_{n}(t) & :=\sup \left\{s e^{2 \mu_{n}(s, r)} \mid r \in \mathbb{R}, t_{0} \leq s \leq t\right\}, \\
S_{n}(t) & :=\sup \left\{\left|E_{n}\right| e^{\lambda_{n}(s, r)} \mid r \in \mathbb{R}, t_{0} \leq s \leq t\right\},
\end{aligned}
$$

we have the following estimate on $\operatorname{supp} f_{n}^{ \pm}(t)$

$$
\sqrt{1+w^{2}+L / t^{2}} \leq \sqrt{1+\left(P_{n}^{ \pm}(t)\right)^{2}+L_{0}^{ \pm} / t_{0}^{2}} \leq C\left(1+L_{0}^{ \pm}\right)\left(1+P_{n}^{ \pm}(t)\right),
$$

so that

$$
\begin{aligned}
\left\|\rho_{n}^{ \pm}(t)\right\|,\left\|p_{n}^{ \pm}(t)\right\| & \leq \frac{C}{t^{2}}\left(1+L_{0}^{ \pm}\right)^{2}\left(1+\left\|\stackrel{\circ}{f^{ \pm}}\right\|\right)\left(1+P_{n}^{ \pm}(t)\right)^{2}\left(1+S_{n-1}(t)\right)^{2} \\
\left\|j_{n}^{ \pm}(t)\right\| & \leq \frac{C}{t}\left(1+L_{0}^{ \pm}\right)^{2}\left(1+\left\|\stackrel{\circ}{f^{ \pm}}\right\|\right)\left(1+P_{n}^{ \pm}(t)\right)^{2},
\end{aligned}
$$

and then using (3.7) and (3.9)

$$
\begin{array}{r}
\left|e^{\mu_{n}-\lambda_{n}} \tilde{\mu}_{n}(t, r)\right|+\left|\dot{\lambda}_{n}(t, r)\right| \leq C(1+\Lambda)\left(1+L_{0}^{ \pm}\right)^{2}\left(1+\left\|f^{ \pm}\right\|\right)\left(1+P_{n}^{ \pm}(t)\right)^{2} \\
\left(1+Q_{n}(t)\right)\left(1+S_{n-1}(t)\right)^{2}
\end{array}
$$

This inequality is used to obtain an estimate on $\operatorname{supp} f_{n+1}^{ \pm}(t)$ for $\left|\dot{W}_{n+1}^{ \pm}\right|$which implies

$$
\begin{gathered}
P_{n+1}^{ \pm}(t) \leq w_{0}^{ \pm}+C^{*} \int_{t_{0}}^{t}\left(1+P_{n}^{ \pm}(s)\right)^{2}\left(1+P_{n+1}^{ \pm}(s)\right)\left(1+Q_{n}(s)\right)^{3 / 2}\left(1+S_{n-1}(s)\right)^{2} \\
\left(1+S_{n}(s)\right) d s
\end{gathered}
$$

with $C^{*}=C(1+\Lambda)\left(1+L_{0}^{ \pm}\right)^{2}\left(1+\left\|\stackrel{\circ}{f^{ \pm}}\right\|\right)$. Setting $\tilde{P}_{n+1}^{ \pm}:=\sup \left\{P_{m}^{ \pm}(t) \mid m \leq n\right\}$, it follows that

$$
\tilde{P}_{n+1}^{ \pm}(t) \leq w_{0}^{ \pm}+C^{*} \int_{t_{0}}^{t}\left(1+\tilde{P}_{n}^{ \pm}(s)\right)^{3}\left(1+Q_{n}(s)\right)^{3 / 2}\left(1+\tilde{S}_{n}(s)\right)^{3} d s,
$$

whence

$$
\tilde{P}_{n+1}(t) \leq w_{0}+C^{*} \int_{t_{0}}^{t}\left(1+\tilde{P}_{n+1}(s)\right)^{3}\left(1+Q_{n}(s)\right)^{3 / 2}\left(1+\tilde{S}_{n}(s)\right)^{3} d s .
$$

Taking the derivative of (3.6) with respect to $t$ leads to

$$
t\left(2 \dot{\mu}_{n} e^{2 \mu_{n}}\right)=8 \pi\left(t e^{2 \mu_{n}}\right)^{2} p_{n}+\frac{k}{t^{2}}\left(t e^{2 \mu_{n}}\right)^{2}+\frac{1}{t} t e^{2 \mu_{n}}-\Lambda\left(t e^{2 \mu_{n}}\right)^{2},
$$


integrating this over $\left[t_{0}, t\right]$ and using integration by parts for the left hand side yields

$$
Q_{n}(t) \leq\left\|t_{0} e^{2 \stackrel{\circ}{\mu}}\right\|+C^{*} \int_{t_{0}}^{t}\left(1+\tilde{P}_{n}(s)\right)\left(1+Q_{n}(s)\right)^{2}\left(1+\tilde{S}_{n-1}(s)\right)^{2} d s .
$$

Next (3.10) implies that

$$
S_{n}(t) \leq\left\|e^{\stackrel{\circ}{\perp}} \underline{\|}\right\|+C^{*} \int_{t_{0}}^{t}\left(1+\tilde{P}_{n}(s)\right)^{2}\left(1+Q_{n}(s)\right)^{1 / 2} d s .
$$

Adding (3.15), (3.16) and (3.17) implies that

$$
\left(\tilde{P}_{n+1}+Q_{n}+\tilde{S}_{n}\right)(t) \leq w_{0}+\left\|t_{0} e^{2 \stackrel{\circ}{\mu}}\right\|+\left\|e^{\stackrel{\circ}{\perp}}\right\|+C^{*} \int_{t_{0}}^{t}\left[1+\left(\tilde{P}_{n+1}+Q_{n}+\tilde{S}_{n}\right)(s)\right]^{15 / 2} d s .
$$

Thus $P_{n}, Q_{n}, S_{n}$ are estimated by $z_{1}(t)$, the right maximal solution of the equation

$$
z_{1}(t) \leq w_{0}+\left\|t_{0} e^{2 \stackrel{\circ}{\mu}}\right\|+\|\stackrel{\circ}{\stackrel{\circ}{\perp}}\|+C^{*} \int_{t_{0}}^{t}\left(1+z_{1}(s)\right)^{15 / 2} d s
$$

which exists on $\left[t_{0}, T^{(1)}\right.$ [, we have $T_{n} \geq T^{(1)}$. Therefore there exists a continuous function $C_{1}(t)$ which depends only on $z_{1}$ as an increasing function such that $\mu_{n}$, $\lambda_{n}, \dot{\lambda}_{n}, \rho_{n}, p_{n}, E_{n}, j_{n}, a_{n}, b_{n}, \tilde{\mu}_{n} e^{\mu_{n}-\lambda_{n}}$ are bounded in the $L^{\infty}$-norm by $C_{1}(t)$.

Next we prove :

Lemma 3.2 The sequences $\mu_{n}^{\prime}, \lambda_{n}^{\prime}, \dot{\lambda}_{n}^{\prime}, \rho_{n}^{\prime}, p_{n}^{\prime}, E_{n}^{\prime}, j_{n}^{\prime}, a_{n}^{\prime}, b_{n}^{\prime}, \tilde{\mu}_{n}^{\prime}$ are uniformly bounded in $n$.

Proof Let us start from equation (3.11). Given Lemma 3.1 most of the quantities on the right hand side of (3.11) can be estimated. The exception is $\mu_{n}^{\prime}$ which is obtained after differentiating (3.6) with respect to $r$ :

$$
\mu_{n}^{\prime}(t, r)=\frac{e^{2 \mu_{n}}}{t}\left(t_{0} \stackrel{\circ}{\mu}(r) e^{-2 \mu \stackrel{\circ}{\mu}}-4 \pi \int_{t}^{t_{0}} s^{2} p_{n}^{\prime}(s, r) d s\right)
$$

again most of the terms are unproblematic with one exception that is $p_{n}^{\prime}$ :

$$
\begin{aligned}
p_{n}^{\prime}(t, r) & =\frac{\pi}{t^{2}} \int_{-\infty}^{\infty} \int_{0}^{\infty} \frac{w^{2}}{\sqrt{1+w^{2}+L / t^{2}}} \partial_{r}\left(f_{n}^{+}+f_{n}^{-}\right)(t, r, w, L) d L d w \\
& -e^{\lambda_{n-1}} E_{n-1} \partial_{r}\left(e^{\lambda_{n-1}} E_{n-1}\right) .
\end{aligned}
$$

Defining

$$
D_{n}(t):=\sup \left\{\left\|\partial_{r} f_{n}^{+}(s)\right\|+\left\|\partial_{r} f_{n}^{-}(s)\right\| \mid t_{0} \leq s \leq t\right\},
$$


and

$$
\Delta_{n}(t):=\sup \left\{\left\|\partial_{r}\left(e^{\lambda_{n}} E_{n}\right)(s)\right\| \mid t_{0} \leq s \leq t\right\},
$$

we deduce the following estimates

$$
\begin{aligned}
\left\|t^{2} p_{n}^{\prime}(t)\right\| & \leq C_{1}(t)\left(D_{n}(t)+\Delta_{n-1}(t)\right) \\
\left\|\mu_{n}^{\prime}(t)\right\| & \leq C_{1}(t)\left(c_{1}+D_{n}(t)+\Delta_{n-1}(t)\right),
\end{aligned}
$$

where $c_{1}:=\left\|e^{-2 \stackrel{\circ}{\mu} \stackrel{\circ}{\mu}^{\prime}}\right\|+\left\|\stackrel{\circ}{\lambda}^{\prime}\right\|+1+|\Lambda|$, and so equation (3.11) implies

$$
\left\|\partial_{r}\left(e^{\lambda_{n}} E_{n}\right)(t)\right\| \leq C_{1}(t)+\int_{t_{0}}^{t} C_{1}(s)\left(c_{1}+D_{n}(s)+\Delta_{n-1}(s)\right) d s
$$

thus

$$
\Delta_{n}(t) \leq C_{1}(t)+\int_{t_{0}}^{t} C_{1}(s)\left(c_{1}+D_{n}(s)+\Delta_{n-1}(s)\right) d s .
$$

Now differentiating (3.7) and (3.8) yields

$$
\begin{aligned}
& \dot{\lambda}_{n}^{\prime}(t, r)=e^{2 \mu_{n}}\left(8 \pi t \mu_{n}^{\prime}(t, r) \rho_{n}(t, r)+4 \pi t \rho_{n}^{\prime}(t, r)-\frac{k}{t} \mu_{n}^{\prime}(t, r)+\Lambda t \mu_{n}^{\prime}\right) \\
& \lambda_{n}^{\prime}(t, r)=\stackrel{\circ}{\lambda}^{\prime}(r)+\int_{t_{0}}^{t} \dot{\lambda}_{n}^{\prime}(s, r) d s,
\end{aligned}
$$

and using the expression for $\rho_{n}^{\prime}$ we obtain

$$
\begin{aligned}
\left\|t \rho_{n}^{\prime}(t)\right\| & \leq C_{1}(t)\left(D_{n}(t)+\Delta_{n-1}(t)\right) \\
\left\|\lambda_{n}^{\prime}(t)\right\|,\left\|\dot{\lambda}_{n}^{\prime}(t)\right\| & \leq C_{1}(t)\left(c_{1}+D_{n}(t)+\Delta_{n-1}(t)\right) .
\end{aligned}
$$

On the other hand it follows from (3.9) that

$$
e^{\mu_{n}-\lambda_{n}} \tilde{\mu}_{n}=-4 \pi t e^{2 \mu_{n}} j_{n}
$$

and

$$
\left|\left(e^{\mu_{n}-\lambda_{n}} \tilde{\mu}_{n}\right)^{\prime}(t, r)\right| \leq C_{1}(t)\left(c_{1}+D_{n}(t)+\Delta_{n-1}(t)\right) .
$$

We can now estimate the derivatives of $G_{n}^{ \pm}$with respect to $r$ and $w$ :

$$
\begin{gathered}
\partial_{r} G_{n}^{ \pm}(t, r, w, L)=\left(\left(\mu_{n}-\lambda_{n}\right)^{\prime} e^{\mu_{n}-\lambda_{n}} \frac{w}{\sqrt{1+w^{2}+L / t^{2}}},\right. \\
\left.-\left(e^{\mu_{n}-\lambda_{n}} \tilde{\mu}_{n}\right)^{\prime} \sqrt{1+w^{2}+L / t^{2}}-\dot{\lambda}_{n}^{\prime} w \pm\left(\mu_{n}^{\prime} e^{\mu_{n}+\lambda_{n}} E_{n}+e^{\mu_{n}} \partial_{r}\left(e^{\lambda_{n}} E_{n}\right)\right)\right), \\
\partial_{w} G_{n}^{ \pm}(t, r, w, L)=\left(e^{\mu_{n}-\lambda_{n}} \frac{1+L / t^{2}}{\left(1+w^{2}+L / t^{2}\right)^{3 / 2}},\right. \\
\left.-e^{\mu_{n}-\lambda_{n}} \tilde{\mu}_{n} \frac{w}{\sqrt{1+w^{2}+L / t^{2}}}-\dot{\lambda}_{n}\right),
\end{gathered}
$$


and thus

$$
\begin{aligned}
\left|\partial_{r} G_{n}^{ \pm}(t, r, w, L)\right| & \leq C_{1}(t)\left(c_{1}+D_{n}(t)+\Delta_{n-1}(t)+\Delta_{n}(t)\right), \\
\left|\partial_{w} G_{n}^{ \pm}(t, r, w, L)\right| & \leq C_{1}(t),
\end{aligned}
$$

for $t \in\left[t_{0}, T^{(1)}\left[, r \in \mathbb{R}, L \in\left[0, L_{0}\right]\right.\right.$ and $|w| \leq z_{1}(t)$. Differentiating the characteristic system with respect to $r$, we obtain

$\frac{d}{d s} \partial_{r}\left(R_{n+1}^{ \pm}, W_{n+1}^{ \pm}\right)(s, t, r, w, L)=\partial_{r} G_{n}^{ \pm}\left(s, R_{n+1}^{ \pm}, W_{n+1}^{ \pm}, L\right) . \partial_{r}\left(R_{n+1}^{ \pm}, W_{n+1}^{ \pm}\right)(s, t, r, w, L)$

it follows that

$$
\begin{aligned}
\left|\frac{d}{d s} \partial_{r}\left(R_{n+1}^{ \pm}, W_{n+1}^{ \pm}\right)(s, t, r, w, L)\right| \leq & C_{1}(s)\left(c_{1}+D_{n}(s)+\Delta_{n-1}(s)+\Delta_{n}(s)\right) \\
& \left|\partial_{r}\left(R_{n+1}^{ \pm}, W_{n+1}^{ \pm}\right)(s, t, r, w, L)\right|
\end{aligned}
$$

therefore by Gronwall's inequality we obtain, for $(r, w, L) \in \operatorname{supp} f_{n+1}^{+}(t) \cup \operatorname{supp} f_{n}^{+}(t) \cup \operatorname{supp} f_{n+1}^{-}(t) \cup \operatorname{supp} f_{n}^{-}(t)$

$$
\left|\partial_{r}\left(R_{n+1}^{ \pm}, W_{n+1}^{ \pm}\right)\left(t_{0}, t, r, w, L\right)\right| \leq \exp \left[\int_{t}^{t_{0}} C_{1}(s)\left(c_{1}+D_{n}(s)+\Delta_{n-1}(s)+\Delta_{n}(s)\right) d s\right]
$$

The definition of $f_{n}^{ \pm}$implies that

$\left\|\partial_{r} f_{n}^{ \pm}(t)\right\| \leq\left\|\partial_{(r, w)} \stackrel{\circ}{f^{ \pm}}\right\| \sup \left\{\left|\partial_{r}\left(R_{n}^{ \pm}, W_{n}^{ \pm}\right)\left(t_{0}, t, r, w, L\right)\right| \mid(r, w, L) \in \operatorname{supp} f_{n}^{+}(t) \cup \operatorname{supp} f_{n}^{-}(t)\right\}$.

Combining this with the previous inequality and using the definition of $D_{n}$ we obtain the following :

$$
D_{n+1}(t) \leq\left(\left\|\partial_{(r, w)} \stackrel{\circ}{f^{+}}\right\|+\left\|\partial_{(r, w)} \stackrel{\circ}{f^{-}}\right\|\right) \exp \left[\int_{t}^{t_{0}} C_{1}(s)\left(c_{1}+D_{n}(s)+\Delta_{n-1}(s)+\Delta_{n}(s)\right) d s\right](3
$$

Let $\tilde{D}_{n}(t):=\sup \left\{D_{m}(t) \mid m \leq n\right\}$ and $\tilde{\Delta}_{n}(t):=\sup \left\{\Delta_{m}(t) \mid m \leq n\right\}$. Then $\left(\tilde{D}_{n}\right)_{n}$ and $\left(\tilde{\Delta}_{n}\right)_{n}$ are increasing, therefore adding (3.18) and (3.19) implies

$$
\begin{aligned}
\tilde{D}_{n+1}(t)+\tilde{\Delta}_{n}(t) \leq & C_{1}(t)+\left(1+\left\|\partial_{(r, w)} f^{+}\right\|+\left\|\partial_{(r, w)} f^{-}\right\|\right) \\
& \exp \left[\int_{t}^{t_{0}} C_{1}(s)\left(c_{1}+\tilde{D}_{n+1}(s)+\tilde{\Delta}_{n}(s)\right) d s\right],
\end{aligned}
$$

hence

$$
\tilde{D}_{n+1}(t)+\tilde{\Delta}_{n}(t) \leq z_{2}(t), t \in\left[t_{0}, T^{(2)}\left[, n \in \mathbb{N},\left[t_{0}, T^{(2)}\left[\subset \left[t_{0}, T^{(1)}[\right.\right.\right.\right.\right.
$$

where $z_{2}$ is the right maximal solution of

$$
z_{2}(t)=C_{1}(t)+\left(1+\left\|\partial_{(r, w)} \stackrel{\circ}{f^{+}}\right\|+\left\|\partial_{(r, w)} \stackrel{\circ}{f^{-}}\right\|\right) \exp \left[\int_{t}^{t_{0}} C_{1}(s)\left(c_{1}+z_{2}(s)\right) d s\right],
$$


which exists on $\left[t_{0}, T^{(2)}\left[\right.\right.$. Therefore there exists a continuous function $C_{2}(t)$ which depends only on $z_{2}$ as an increasing function such that all the quantities estimated against $D_{n}$ and $\Delta_{n}$ are bounded in the $L^{\infty}$-norm by $C_{2}(t)$.

The following lemma deals with convergence of iterates.

Lemma 3.3 The sequences $f_{n}^{+}, f_{n}^{-}, \lambda_{n}, \mu_{n}, E_{n}, \dot{\lambda}_{n}, \dot{\mu}_{n}, \tilde{\mu}_{n}, \rho_{n}, p_{n}, j_{n}, a_{n}, b_{n}$ converge uniformly on an arbitrary compact subset $\left[t_{0}, T^{(3)}\right] \subset\left[t_{0}, T^{(2)}\right]$ on which the previous estimates hold.

Proof Define for $t \in\left[t_{0}, T^{(3)}\right]$

$$
\begin{array}{r}
\alpha_{n}(t):=\sup \left\{\left\|\left(f_{n+1}^{+}-f_{n}^{+}\right)(s)\right\|+\left\|\left(f_{n+1}^{-}-f_{n}^{-}\right)(s)\right\|+\left\|\left(\lambda_{n+1}-\lambda_{n}\right)(s)\right\|\right. \\
\left.+\left\|\left(\mu_{n+1}-\mu_{n}\right)(s)\right\|+\left\|\left(e^{\lambda_{n}} E_{n}-e^{\lambda_{n-1}} E_{n-1}\right)(s)\right\| ; t_{0} \leq s \leq t\right\}
\end{array}
$$

and let $C$ denote a constant which may depend on the functions $z_{1}$ and $z_{2}$ introduced previously. Then using Lemma3.1 we have

$$
\begin{array}{r}
\left\|\rho_{n+1}(t)-\rho_{n}(t)\right\|,\left\|\rho_{n+1}(t)-\rho_{n}(t)\right\|,\left\|p_{n+1}(t)-p_{n}(t)\right\|, \\
\left\|j_{n+1}(t)-j_{n}(t)\right\|,\left\|a_{n+1}(t)-a_{n}(t)\right\|,\left\|b_{n+1}(t)-b_{n}(t)\right\| \leq C \alpha_{n}(t) .
\end{array}
$$

Using mean value theorem to estimate differences $e^{\lambda_{n+1}+\mu_{n+1}}-e^{\lambda_{n}+\mu_{n}}$ and $e^{\mu_{n+1}}-$ $e^{\mu_{n}}$, we deduce from (3.7) and (3.9) that

$$
\left\|\dot{\lambda}_{n+1}(t)-\dot{\lambda}_{n}(t)\right\|,\left\|\tilde{\mu}_{n+1}(t)-\tilde{\mu}_{n}(t)\right\| \leq C \alpha_{n}(t),
$$

(3.8) thus implies

$$
\left\|\left(\lambda_{n+1}-\lambda_{n}\right)(t)\right\| \leq C \int_{t_{0}}^{t} \alpha_{n}(s) d s .
$$

By mean value theorem (3.6) implies

$$
\left\|\left(\mu_{n+1}-\mu_{n}\right)(t)\right\| \leq C \int_{t_{0}}^{t} \alpha_{n}(s) d s .
$$

Following step 3 in the proof of [6, thm 3.1] we have

$$
\left|(R, W)_{n+1}^{+}-(R, W)_{n}^{+}\right|\left(t_{0}, t, r, w, L\right),\left|(R, W)_{n+1}^{-}-(R, W)_{n}^{-}\right|\left(t_{0}, t, r, w, L\right) \leq C \int_{t_{0}}^{t} \alpha_{n-1}(s) d s
$$

which implies, using the fact that $f_{n}^{ \pm}$was defined in terms of the characteristics, and by mean value theorem

$$
\left\|\left(f_{n+1}^{+}-f_{n}^{+}\right)(t)\right\|,\left\|\left(f_{n+1}^{-}-f_{n}^{-}\right)(t)\right\| \leq C \int_{t_{0}}^{t} \alpha_{n-1}(s) d s .
$$

From (3.10) we deduce that

$$
\left\|\left(e^{\lambda_{n}} E_{n}-e^{\lambda_{n-1}} E_{n-1}\right)(t)\right\| \leq C \int_{t_{0}}^{t} \alpha_{n-1}(s) d s .
$$


Adding (3.20)-(3.23) gives

$$
\alpha_{n}(t) \leq C \int_{t_{0}}^{t}\left(\alpha_{n}(s)+\alpha_{n-1}(s)\right) d s,
$$

then by Gromwall's inequality

$$
\alpha_{n}(t) \leq C \int_{t_{0}}^{t} \alpha_{n-1}(s) d s
$$

and by induction

$$
\alpha_{n}(t) \leq \frac{C^{n+1}}{n !}, \text { for } n \in \mathbb{N}, t \in\left[t_{0}, T^{(3)}\right]
$$

We then conclude the uniform convergence of the iterates. In $L^{\infty}$-norm, $\lambda_{n} \rightarrow \lambda$, $\mu_{n} \rightarrow \mu, \tilde{\mu}_{n} \rightarrow \tilde{\mu}, f_{n}^{+} \rightarrow f^{+}, f_{n}^{-} \rightarrow f^{-}, E_{n} \rightarrow E$.

In order to prove that the latter limits are regular in the sense of Definition 2.1 we need to show the uniform convergence of the derivatives of the iterates above.

Lemma 3.4 The sequences $\partial_{r} f_{n}^{+}, \partial_{w} f_{n}^{+}, \partial_{r} f_{n}^{-}, \partial_{w} f_{n}^{-}, \lambda_{n}^{\prime}, \mu_{n}^{\prime}, \tilde{\mu}_{n}^{\prime}, \partial_{r}\left(e^{\lambda_{n}} E_{n}\right)$ are uniformly convergent.

Proof Fix $T^{(4)} \in\left[t_{0}, T^{(2)}\right], t \in\left[t_{0}, T^{(4)}\right],|w|<U, L<\min \left(L_{0}^{+}, L_{0}^{-}\right), t_{0} \leq s \leq t$. For $\partial \in\left\{\partial_{r}, \partial_{w}\right\}$ and $s \mapsto\left(R_{n}^{ \pm}(s), W_{n}^{ \pm}(s)\right)$ the solution of the characteristic system associated to equation (3.5) in $f_{n}^{ \pm}$, define

$$
\begin{gathered}
\xi_{n}^{ \pm}(s):=e^{\left(\lambda_{n}-\mu_{n}\right)\left(s, R_{n}^{ \pm}\right)} \partial R_{n}^{ \pm}(s, t, r, w, L), \\
\eta_{n}^{ \pm}(s):=\partial W_{n}^{ \pm}(s, t, r, w, L) \\
+\left.\left(\sqrt{1+w^{2}+L / s^{2}} e^{\lambda_{n}-\mu_{n}} \dot{\lambda}_{n}\right)\right|_{\left(s,\left(R_{n}^{ \pm}, W_{n}^{ \pm}\right)(s, t, r, w, L)\right)} \partial R_{n}^{ \pm}(s, t, r, w, L) .
\end{gathered}
$$

Reasoning as in step 4 for the proof of theorem 3.1 in [6] it can be proved with minor changes that for all positive real number $\epsilon$, there is a non negative integer $N$ such that for $n>N$,

$$
\begin{aligned}
\left(\left|\xi_{n+1}^{ \pm}-\xi_{n}^{ \pm}\right|+\left|\eta_{n+1}^{ \pm}-\eta_{n}^{ \pm}\right|\right)(s) & \leq C \epsilon \\
& +C \int_{t_{0}}^{s}\left(\left|\xi_{n+1}^{ \pm}-\xi_{n}^{ \pm}\right|+\left|\eta_{n+1}^{ \pm}-\eta_{n}^{ \pm}\right|\right)(\tau) d \tau
\end{aligned}
$$

the coefficients $a_{n, i}$ being as in [6] except $a_{n, 3}$ which is replaced here by

$$
\begin{aligned}
a_{n, 3}^{ \pm}(s, r, w, L) & :=-\frac{1}{s} \sqrt{1+w^{2}+L / s^{2}}\left(\dot{\lambda}_{n}-\dot{\mu}_{n}+\frac{L / s^{2}}{1+w^{2}+L / s^{2}} \dot{\lambda}_{n}\right) \\
& \pm e^{2 \mu_{n}}\left(\mu_{n}^{\prime} E_{n}+e^{-\lambda_{n}} \partial_{r}\left(e^{\lambda_{n}} E_{n}\right)\right) .
\end{aligned}
$$


By Gronwall's lemma it follows that the sequences $\xi_{n}^{ \pm}$and $\eta_{n}^{ \pm}$converge uniformly. The transformation from $\left(\partial R_{n}^{ \pm}, \partial W_{n}^{ \pm}\right)$to $\left(\xi_{n}^{ \pm}, \eta_{n}^{ \pm}\right)$being invertible with convergent coefficients, we deduce the convergence of $\partial_{r, w}\left(R_{n}^{ \pm}, W_{n}^{ \pm}\right)$and thus the convergence of $\partial_{r} f_{n}^{ \pm}$and $\partial_{w} f_{n}^{ \pm}$, using the fact that $f_{n}^{ \pm}$was defined in terms of characteristics. Let us prove the convergence of $\lambda_{n}^{\prime}, \mu_{n}^{\prime}, \tilde{\mu}_{n}^{\prime}, \partial_{r}\left(e^{\lambda_{n}} E_{n}\right)$. Define

$$
\begin{aligned}
\gamma_{n}(t) & :=\sup \left\{\left|\xi_{n+1}^{+}-\xi_{n}^{+}\right|(s)+\left|\xi_{n+1}^{-}-\xi_{n}^{-}\right|(s)+\left|\eta_{n+1}^{+}-\eta_{n}^{+}\right|(s)\right. \\
& +\left|\eta_{n+1}^{-}-\eta_{n}^{-}\right|(s)+\left\|\left(\mu_{n+1}^{\prime}-\mu_{n}^{\prime}\right)(s)\right\|+\left\|\left(\lambda_{n+1}^{\prime}-\lambda_{n}^{\prime}\right)(s)\right\| \\
& \left.+\left\|\partial_{r}\left(e^{\lambda_{n+1}} E_{n+1}\right)-\partial_{r}\left(e^{\lambda_{n}} E_{n}\right)\right\|(s), t_{0} \leq s \leq t\right\} .
\end{aligned}
$$

The sequences $\mu_{n}, \dot{\mu}_{n}, \tilde{\mu}_{n}, \dot{\lambda}_{n}, e^{\lambda_{n}} E_{n}, \rho_{n}, j_{n}, a_{n}, b_{n}$ converge uniformly, we then take the above integer $N$ large enough so that we have for $n>N$,

$$
\begin{aligned}
& \left\|\left(e^{\lambda_{n}} E_{n}-e^{\lambda_{n-1}} E_{n-1}\right)(s)\right\|,\left\|\left(\mu_{n+1}-\mu_{n}\right)(s)\right\|,\left\|\left(\tilde{\mu}_{n}-\tilde{\mu}_{n-1}\right)(s)\right\|, \\
& \quad\left\|\left(\dot{\lambda}_{n+1}-\dot{\lambda}_{n}\right)(s)\right\|,\left\|\left(\rho_{n+1}-\rho_{n}\right)(s)\right\|,\left\|\left(j_{n+1}-j_{n}\right)(s)\right\|, \\
& \quad\left\|\left(\dot{\mu}_{n+1}-\dot{\mu}_{n}\right)(s)\right\|,\left\|\left(a_{n+1}-a_{n}\right)(s)\right\|,\left\|\left(b_{n+1}-b_{n}\right)(s)\right\| \leq \epsilon .
\end{aligned}
$$

Taking $\partial=\partial_{r}$, it follows from (3.24)-(3.25) that

$$
\begin{gathered}
\partial R_{n}^{ \pm}(s):=e^{\left(\mu_{n}-\lambda_{n}\right)\left(s, R_{n}^{ \pm}\right)} \xi_{n}^{ \pm}(s), \\
\partial W_{n}^{ \pm}(s)=\eta_{n}^{ \pm}(s)-\left(\sqrt{1+w^{2}+L / s^{2}} \dot{\lambda}_{n}\right) \xi_{n}^{ \pm}(s) .
\end{gathered}
$$

Using these equations and since $f_{n}^{ \pm}$was defined in terms of characteristics we obtain

$$
\begin{aligned}
\left\|\left(\partial_{r} f_{n+1}^{ \pm}-\partial_{r} f_{n}^{ \pm}\right)(s)\right\| & \leq\left\|\partial_{r, w} f^{ \pm}\right\|\left(\left|\partial_{r} R_{n+1}^{ \pm}-\partial_{r} R_{n}^{ \pm}\right|+\left|\partial_{r} W_{n+1}^{ \pm}-\partial_{r} W_{n}^{ \pm}\right|\right)(s) \\
& \leq\left\|\partial_{r, w} f^{ \pm}\right\|\left(\left|e^{\mu_{n+1}-\lambda_{n+1}} \xi_{n+1}^{ \pm}-e^{\mu_{n}-\lambda_{n}} \xi_{n}^{ \pm}\right|\right. \\
& \left.+\left|\eta_{n+1}^{ \pm}-\eta_{n}^{ \pm}\right|+\left|\dot{\lambda}_{n+1} \xi_{n+1}^{ \pm}-\dot{\lambda}_{n} \xi_{n}^{ \pm}\right|\right)(s)
\end{aligned}
$$

this implies, using (3.28) and the fact that $\lambda_{n}, \mu_{n}, \dot{\lambda}_{n}, \xi_{n}$ are bounded:

$$
\left\|\left(\partial_{r} f_{n+1}^{ \pm}-\partial_{r} f_{n}^{ \pm}\right)(s)\right\| \leq C \epsilon+C\left(\left|\xi_{n+1}^{ \pm}-\xi_{n}^{ \pm}\right|+\left|\eta_{n+1}^{ \pm}-\eta_{n}^{ \pm}\right|\right)(s) .
$$

Using (3.27), (3.28), 3.32) and the expressions of $p_{n}, \rho_{n}, j_{n}$, we deduce that

$\left\|\left(\rho_{n+1}^{\prime}-\rho_{n}^{\prime}\right)(s)\right\|,\left\|\left(p_{n+1}^{\prime}-p_{n}^{\prime}\right)(s)\right\|,\left\|\left(j_{n+1}^{\prime}-j_{n}^{\prime}\right)(s)\right\| \leq C \epsilon+C\left(\gamma_{n}+\gamma_{n-1}\right)(s)$.

Now taking the derivative of (3.6) with respect to $r$, using (3.28), (3.33) and the fact that $\mu_{n}, \mu_{n}^{\prime}$ are bounded we deduce an estimate for $\mu_{n}^{\prime}$ :

$$
\left\|\left(\mu_{n+1}^{\prime}-\mu_{n}^{\prime}\right)(s)\right\| \leq C \epsilon+C \int_{t_{0}}^{s}\left(\gamma_{n}+\gamma_{n-1}\right)(\tau) d \tau .
$$


For $\lambda_{n}^{\prime}$, we first take the derivative of (3.7) with respect to $r$ and obtain

$$
\dot{\lambda}_{n}^{\prime}=\left(8 \pi t \mu_{n}^{\prime} \rho_{n}+4 \pi t \rho_{n}^{\prime}\right) e^{2 \mu_{n}}+(\Lambda t-k / t) \mu_{n}^{\prime} e^{2 \mu_{n}},
$$

this shows that $\dot{\lambda}_{n}^{\prime}$ is bounded. Subtracting (3.35) written for $n+1$ and $n$, we obtain the following, using the fact that $\rho_{n}, \mu_{n}, \mu_{n}^{\prime}$ are bounded and (3.27), (3.28) and (3.32):

$$
\left\|\left(\dot{\lambda}_{n+1}^{\prime}-\dot{\lambda}_{n}^{\prime}\right)(s)\right\| \leq C \epsilon+C\left(\gamma_{n}+\gamma_{n-1}\right)(s),
$$

and integrating this over $\left[t_{0}, t\right]$ it follows from (3.8) that

$$
\left\|\left(\lambda_{n+1}^{\prime}-\lambda_{n}^{\prime}\right)(s)\right\| \leq C \epsilon+C \int_{t_{0}}^{t}\left(\gamma_{n}+\gamma_{n-1}\right)(\tau) d \tau .
$$

For $\tilde{\mu}_{n}^{\prime}$, we take the derivative of (3.9) with respect to $r$, subtract the expressions written for $n+1$ and $n$, and use (3.27), (3.28) and (3.32) to obtain

$$
\left\|\left(\tilde{\mu}_{n+1}^{\prime}-\tilde{\mu}_{n}^{\prime}\right)(s)\right\| \leq C \epsilon+C\left(\gamma_{n}+\gamma_{n-1}\right)(s) .
$$

Now for an estimate for $\partial_{r}\left(e^{\lambda_{n}} E_{n}\right)$, we subtract the expressions written for $n+1$ and $n$ from (3.11), use (3.27), (3.28), (3.32) and the fact that $\lambda_{n}, \mu_{n}, \mu_{n}^{\prime}, a_{n}, b_{n}$, $\tilde{\mu}_{n}$ are bounded to obtain

$$
\left\|\left(\partial_{r}\left(e^{\lambda_{n+1}} E_{n+1}\right)-\partial_{r}\left(e^{\lambda_{n}} E_{n}\right)\right)(s)\right\| \leq C \epsilon+C \int_{t_{0}}^{t}\left(\gamma_{n}+\gamma_{n-1}\right)(\tau) d \tau .
$$

Now we can add (3.26), (3.34), (3.37) and (3.39) to obtain, after taking the supremum over $s \in\left[t_{0}, t\right]$ :

$$
\gamma_{n}(t) \leq C \epsilon+C \int_{t_{0}}^{t}\left(\gamma_{n}+\gamma_{n-1}\right)(s) d s
$$

and setting $\tilde{\gamma}_{n}(t)=\sup \left\{\gamma_{m}, m \leq n\right\}$, we deduce by Gronwall's lemma that

$$
\tilde{\gamma}_{n}(t) \leq C \epsilon, n>N, t \in\left[t_{0}, T^{(4)}\right] .
$$

Thus the sequence $\tilde{\gamma}_{n}$ converges uniformly to 0. By (3.34), (3.37), (3.38) and (3.39), the sequences $\tilde{\mu}_{n}^{\prime}, \mu_{n}^{\prime}, \lambda_{n}^{\prime}$ and $\partial_{r}\left(e^{\lambda_{n}} E_{n}\right)$ then converge uniformly on $\left[t_{0}, T^{(4)}\right]$.

The regularity of $f^{+}, f^{-}, \lambda, \mu, \tilde{\mu}$ and $E$ follows. Using the convergence of the derivatives, it can be prove as in [6] that $\left(f^{+}, f^{-}, \lambda, \mu, \tilde{\mu}, E\right)$ is a regular solution of (3.1), (3.2), (1.14), (1.15), (3.3), (1.19) and then $\left(f^{+}, f^{-}, \lambda, \mu, E\right)$ solves (1.12)(1.19). To end the proof of Theorem 1.1 we prove the uniqueness of the solution. Let $\left(f_{i}^{+}, f_{i}^{-}, \lambda_{i}, \mu_{i}, E_{i}\right), i=1,2$ be two regular solutions of the Cauchy problem for the same initial data $\left(\stackrel{\circ}{f^{+}}, \stackrel{\circ}{f^{-}}, \stackrel{\circ}{\lambda}, \stackrel{\circ}{\mu}, \stackrel{\circ}{E}\right)$ at $t=t_{0}$. Setting

$$
\begin{array}{r}
\alpha(t):=\sup \left\{\left\|\left(f_{1}^{+}-f_{2}^{+}\right)(s)\right\|+\left\|\left(f_{1}^{-}-f_{2}^{-}\right)(s)\right\|+\left\|\left(\lambda_{1}-\lambda_{2}\right)(s)\right\|\right. \\
\left.+\left\|\left(\mu_{1}-\mu_{2}\right)(s)\right\|+\left\|\left(e^{\lambda_{1}} E_{1}-e^{\lambda_{2}} E_{2}\right)(s)\right\| ; t_{0} \leq s \leq t\right\},
\end{array}
$$


and proceeding similarly as to prove the convergence of iterates leads to

$$
\alpha(t) \leq C \int_{t_{0}}^{t} \alpha(s) d s
$$

which implies that $\alpha(t)=0$ for $t \in\left[t_{0}, \infty[\right.$. This proves uniqueness and completes the proof of Theorem 1.1

\subsection{Proof of Theorem 1.2}

Let $\left(f^{+}, f^{-}, \lambda, \mu, \tilde{\mu}, E\right)$ be a right maximal solution of the auxiliary system (3.1), (3.2), (1.14), (1.15), (3.3), (1.19) with existence interval $\left[t_{0}, T_{\max }[\right.$. Then we have seen that $\left(f^{+}, f^{-}, \lambda, \mu, E\right)$ solves (1.12)-(1.19). By assumption

$$
\begin{aligned}
Q_{*} & :=\sup \left\{t e^{2 \mu(t, r)} \mid r \in \mathbb{R}, t_{0} \leq t<T_{\max }\right\}<\infty, \\
S_{*} & :=\sup \left\{|E| e^{\lambda(t, r)} \mid r \in \mathbb{R}, t_{0} \leq t<T_{\max }\right\}<\infty,
\end{aligned}
$$

and $P_{*}<\infty$ where $P_{*}:=P_{*}^{+}+P_{*}^{-}$with

$$
P_{*}^{ \pm}:=\sup \left\{|w|,(r, w, L) \in \operatorname{supp} f^{ \pm}(t), t \in\left[t_{0}, T_{\max }[\} .\right.\right.
$$

We assume that $T_{\max }<\infty$ and take $\left.t_{1} \in\right] t_{0}, T_{\max }[$. We will show that the system has a solution with initial data $\left(f^{+}\left(t_{1}\right), f^{-}\left(t_{1}\right), \lambda\left(t_{1}\right), \mu\left(t_{1}\right), E\left(t_{1}\right)\right)$ prescribed at $t=t_{1}$ which exists on an interval $\left[t_{1}, t_{1}+\delta\right]$ with $\delta>0$ independent of $t_{1}$. By moving $t_{1}$ close enough to $T_{\max }$ this would extend the initial solution beyond $T_{\max }$, a contradiction to the initial solution being right maximal. We have proved previously that such a solution exists at least on the right maximal existence interval of the solutions $z_{1}, z_{2}$ of

$$
\begin{gathered}
z_{1}(t)=W\left(t_{1}\right)+\left\|t_{1} e^{2 \mu\left(t_{1}\right)}\right\|+\left\|e^{\lambda\left(t_{1}\right)} E\left(t_{1}\right)\right\|+C^{*} \int_{t_{0}}^{t}\left(1+z_{1}(s)\right)^{15 / 2} d s, \\
z_{2}(t)=C_{1}(t)+\left(1+\left\|\partial_{(r, w)} f^{+}\left(t_{1}\right)\right\|+\left\|\partial_{(r, w)} f^{-}\left(t_{1}\right)\right\|\right) \exp \left[\int_{t}^{t_{0}} C_{1}(s)\left(c_{1}+z_{2}(s)\right) d s\right],
\end{gathered}
$$

where $W\left(t_{1}\right):=W^{+}\left(t_{1}\right)+W^{-}\left(t_{1}\right)$,

$$
\begin{aligned}
W^{ \pm}\left(t_{1}\right) & :=\sup \left\{|w|,(r, w, L) \in \operatorname{supp} f^{ \pm}\left(t_{1}\right)\right\}, \\
C^{*} & =C(1+\Lambda)\left(1+L_{0}\right)^{2}\left(1+\left\|f^{+}\left(t_{1}\right)\right\|+\left\|f^{-}\left(t_{1}\right)\right\|\right), \\
c_{1} & :=1+\Lambda+\left\|\lambda^{\prime}\left(t_{1}\right)\right\|+\left\|e^{-2 \mu\left(t_{1}\right)} \mu^{\prime}\left(t_{1}\right)\right\|,
\end{aligned}
$$

and $C_{1}$ is an increasing function of $z_{1}$. Now $W\left(t_{1}\right) \leq P_{*},\left\|t_{1} e^{2 \mu\left(t_{1}\right)}\right\| \leq Q_{*}$, $\left\|e^{\lambda\left(t_{1}\right)} E\left(t_{1}\right)\right\| \leq S_{*},\left\|f^{ \pm}\left(t_{1}\right)\right\|=\left\|\stackrel{\circ}{f^{ \pm}}\right\|, L_{0}$ is unchanged. Thus we have uniform 
bounds $W\left(t_{1}\right)+\left\|t_{1} e^{2 \mu\left(t_{1}\right)}\right\|+\left\|e^{\lambda\left(t_{1}\right)} E\left(t_{1}\right)\right\| \leq M_{1}, C^{*} \leq M_{2}$. On the other hand we can use the expressions for $\lambda^{\prime}, \mu^{\prime}, \dot{\lambda}^{\prime}$, some estimates proved in lemmas 3.1, 3.2 , and 3.3 to obtain uniform bounds $c_{1} \leq M_{3}, C_{1}(t)+\left(1+\left\|\partial_{(r, w)} f^{+}\left(t_{1}\right)\right\|+\|\right.$ $\left.\partial_{(r, w)} f^{-}\left(t_{1}\right) \|\right) \leq M_{4}$. Let $y_{1}$ and $y_{2}$ be the right maximal solution of

$$
\begin{aligned}
& y_{1}(t)=M_{1}+M_{2} \int_{t_{1}}^{t}\left(1+y_{1}(s)\right)^{15 / 2} d s, \\
& y_{2}(t)=M_{4} \exp \left[\int_{t_{1}}^{t} C_{1}^{*}(s)\left(M_{3}+y_{2}(s)\right) d s\right],
\end{aligned}
$$

respectively, where $C_{1}^{*}$ depends on $y_{1}$ in the same way as $C_{1}$ depends on $z_{1}$. Then $y_{1}$ and $y_{2}$ exist on an interval $\left[t_{1}, t_{1}+\delta\right]$ with $\delta>0$ independent of $t_{1}$. If we choose $t_{1}$ such that $T_{\max }<t_{1}+\delta$ then $z_{1} \leq y_{1}, z_{2} \leq y_{2}$, in particular $z_{1}$ and $z_{2}$ exist on $\left[t_{1}, t_{1}+\delta\right]$. This completes the proof of theorem 1.2

\subsection{Proof of Proposition 1.3}

We start by proving that the boundedness of the lapse function $\mu$ implies that of $e^{\lambda} E$. This follows by integrating (1.19) with respect to $t$, and using the fact that $|b| \leq \bar{a}, \bar{a}$ being similar to $a$ with $f^{+}-f^{-}$replaced by $f^{+}+f^{-}$, and $\bar{a}$ is bounded.

Next we show how to obtain the bound on $w$. Since we are in the non-vacuum case one has $w_{0}^{ \pm}>0$ and $L_{0}^{ \pm}>0$. For $t \geq t_{0}$ define

$$
\begin{aligned}
& P_{+}^{ \pm}(t):=\max \left\{0, \max \left\{w \mid(r, w, L) \in \operatorname{supp} f^{ \pm}(t)\right\}\right\}, \\
& P_{-}^{ \pm}(t):=\min \left\{0, \min \left\{w \mid(r, w, L) \in \operatorname{supp} f^{ \pm}(t)\right\}\right\} .
\end{aligned}
$$

Let $(r(s), w(s), L)$ be a characteristic curve in the support of $f^{ \pm}$. Assume that $P_{+}^{ \pm}(t)>0$ for some $t \in\left[t_{0}, T_{\max }[\right.$, and let $w(t)>0$. We have

$$
\begin{aligned}
\dot{w}(s) & =-\dot{\lambda} w-e^{\mu-\lambda} \mu^{\prime} \sqrt{1+w^{2}+L / s^{2}} \mp e^{\lambda+\mu} E \\
& =\frac{4 \pi^{2}}{s} e^{2 \mu} \int_{-\infty}^{\infty} \int_{0}^{\infty}\left(\tilde{w} \sqrt{1+w^{2}+L / s^{2}}-w \sqrt{1+\tilde{w}^{2}+\tilde{L} / s^{2}}\right)\left(f^{+}+f^{-}\right) d \tilde{L} d \tilde{w} \\
& \mp e^{\lambda+\mu} E+\frac{1}{2 s} w+\frac{\left(k-\Lambda s^{2}\right) e^{2 \mu}}{2 s} w-2 \pi s e^{2 \mu}\left(e^{2 \lambda} E^{2}+c s^{-4}\right) w .
\end{aligned}
$$

As long as $w(s)>0$ we have the following estimates: if $\tilde{w} \leq 0$ then $\tilde{w} \sqrt{1+w^{2}+L / s^{2}}-w \sqrt{1+\tilde{w}^{2}+\tilde{L} / s^{2}} \leq 0$; if $\tilde{w}>0$ then we drop the last two terms of the right hand side in (3.41) since they are negative, and then obtain

$$
\begin{aligned}
\dot{w}(s) & \leq \frac{C}{s} \int_{0}^{P_{+}^{ \pm}(s)} \int_{0}^{L_{0}} \frac{\tilde{w}(1+L)}{w}\left(f^{+}+f^{-}\right) d \tilde{L} d \tilde{w}+\frac{w}{s}+C \\
& \leq \frac{C}{s}\left[\frac{\left(P_{+}^{ \pm}(s)\right)^{2}}{w(s)}+w(s)+s\right]
\end{aligned}
$$


that is

$$
\frac{d}{d s} w(s)^{2} \leq \frac{C}{s}\left(P_{+}^{ \pm}(s)\right)^{2}+C P_{+}^{ \pm}(s) .
$$

Letting $t_{1} \in\left[t_{0}, t\left[\right.\right.$ be defined minimal such that $w(s)>0$ for $s \in\left[t_{1}, t[\right.$, we obtain

$$
w^{2}(t) \leq w^{2}\left(t_{1}\right)+C \int_{t_{1}}^{t}\left[s^{-1}\left(P_{+}^{ \pm}(s)\right)^{2}+P_{+}^{ \pm}(s)\right] d s .
$$

If $t_{1}=t_{0}$ then $w\left(t_{1}\right) \leq w_{0}$, otherwise $w\left(t_{1}\right)=0$. In any case it follows that

$$
w^{2}(t) \leq w_{0}^{2}+C \int_{t_{1}}^{t}\left[s^{-1}\left(P_{+}^{ \pm}(s)\right)^{2}+P_{+}^{ \pm}(s)\right] d s .
$$

Thus

$$
\left(P_{+}^{ \pm}(t)\right)^{2} \leq\left(w_{0}^{2}+C t\right)+C \int_{t_{1}}^{t}\left(s^{-1}+1\right)\left(P_{+}^{ \pm}(s)\right)^{2} d s, \text { for } \mathrm{t} \in\left[\mathrm{t}_{0}, \mathrm{~T}_{\max }[.\right.
$$

By Gronwall's inequality it follows that $P_{+}^{ \pm}$is bounded on $\left[t_{0}, T_{\max }[\right.$.

Estimating $\dot{w}(s)$ from below in the case $w(s)<0$ along the same lines shows that $P_{-}^{ \pm}$is bounded as well.

\subsection{Proof of Theorem 1.4}

We prove that $\mu$ is bounded on $\left[t_{0}, T_{\max }\right)$.

A lengthy computation leads to

$$
\begin{aligned}
\frac{d}{d t} \int_{0}^{1} e^{\mu+\lambda} \rho(t, r) d r & =-\frac{1}{t} \int_{0}^{1} e^{\mu+\lambda}\left[2 \rho+q-\frac{\rho+p}{2}\left[1+\left(k-\Lambda t^{2}\right) e^{2 \mu}\right]\right] d r \\
& \leq-\frac{2}{t} \int_{0}^{1} e^{\mu+\lambda} \rho d r+\frac{1}{t} \int_{0}^{1} e^{\mu+\lambda} \frac{\rho+p}{2}\left[1+\left(k-\Lambda t^{2}\right) e^{2 \mu}\right] d r
\end{aligned}
$$

since $q$ is nonnegative. Using the fact that $\rho+p \geq 0, \rho \geq p$ and $k-\Lambda t^{2} \leq 0$ it follows that

$$
\frac{d}{d t} \int_{0}^{1} e^{\mu+\lambda} \rho(t, r) d r \leq-\frac{1}{t} \int_{0}^{1} e^{\mu+\lambda} \rho d r
$$

and by Gronwall's inequality

$$
\int_{0}^{1} e^{\mu+\lambda} \rho(t, r) d r \leq C t^{-1} t \in\left[t_{0}, T_{\max }\right) .
$$

On the other hand using the equation $\mu^{\prime}=-4 \pi t e^{\mu+\lambda} j$, 3.44) and the fact that $|j| \leq \rho$ we find

$$
\left|\mu(t, r)-\int_{0}^{1} \mu(t, \sigma) d \sigma\right| \leq C, t \in\left[t_{0}, T_{\max }\right), r \in[0,1] .
$$


Next using (1.15), $p-\rho \leq 0$ and $k-\Lambda t^{2} \leq 0$, we have

$$
\begin{aligned}
\frac{\partial}{\partial t} e^{\mu-\lambda} & =e^{\mu-\lambda}\left[4 \pi t e^{2 \mu}(p-\rho)+\frac{1+k e^{2 \mu}}{t}-\Lambda t e^{2 \mu}\right] \\
& \leq e^{\mu-\lambda}\left[\frac{1}{t}+\frac{k-\Lambda t^{2}}{t} e^{2 \mu}\right] \\
& \leq \frac{1}{t} e^{\mu-\lambda}
\end{aligned}
$$

so that

$$
e^{(\mu-\lambda)(t, r)} \leq C t, t \in\left[t_{0}, T_{\max }, r \in[0,1] .\right.
$$

Now using $p-\rho \leq 0$ and $k-\Lambda t^{2} \leq 0$, it follows that

$$
\begin{aligned}
\int_{0}^{1} \mu(t, r) d r & =\int_{0}^{1} \stackrel{\circ}{\mu}(r) d r+\int_{t_{0}}^{t} \int_{0}^{1} \dot{\mu}(s, r) d r d s \\
& \leq C+\int_{t_{0}}^{t} \frac{1}{2 s} \int_{0}^{1}\left[e^{2 \mu}\left(8 \pi s^{2} p+k-\Lambda s^{2}\right)+1\right] d r d s \\
& \leq C+\int_{t_{0}}^{t} \frac{1}{2 s} \int_{0}^{1}\left(8 \pi s^{2} e^{\mu-\lambda} e^{\mu+\lambda} \rho+1\right) d r d s \\
& \leq C+\frac{1}{2} \ln \left(t / t_{0}\right)+C \int_{t_{0}}^{t} s^{2} \int_{0}^{1} e^{\mu+\lambda} \rho d r d s \\
& \leq C+\frac{1}{2} \ln \left(t / t_{0}\right)+C t^{2},
\end{aligned}
$$

and using (3.45) we obtain

$$
\mu(t, r) \leq C\left(1+t^{2}+\ln t\right), t \in\left[t_{0}, T_{\max }\right) r \in[0,1] .
$$

$\mu$ is then bounded on $\left[t_{0}, T_{\max }\right)$ and by proposition 1.3 the proof of theorem 1.4 is complete.

\subsection{Proof of Theorem 1.5}

The equation of motion for charged particles is given by the following differential system for a path $\nu \mapsto\left(\tau, v^{0}, v^{i}\right)(\nu)$ :

$$
\frac{d \tau}{d \nu}=v^{0}, \frac{d v^{0}}{d \nu}=k_{i j} v^{i} v^{j}, \frac{d v^{i}}{d \nu}=2 k_{j}^{i} v^{j} v^{0}-\gamma_{m n}^{i} v^{m} v^{n} \mp\left(F_{0}{ }^{i} v^{0}+F_{j}{ }^{i} v^{j}\right) .
$$

For a particle with rest mass $m$ moving forward in time, $v^{0}=\left(m^{2}+g_{i j} v^{i} v^{j}\right)^{1 / 2}$. Then the relation between coordinate time $\tau$ and proper time $\nu$ is

$$
\frac{d \tau}{d \nu}=\left(m^{2}+g_{i j} v^{i} v^{j}\right)^{1 / 2}
$$


In order to prove the completeness of trajectories it is useful to control $g_{i j} v^{i} v^{j}$ as a function of $\tau$. As in [4, we can define, from the Vlasov equations (1.33)-(1.34), the characteristic curve $V^{i}(\tau)$ satisfying

$\frac{d V_{i}}{d \tau}=-\left(1+g_{r s} V^{r} V^{s}\right)^{-1 / 2} \gamma_{m n}^{j} V_{p} V_{q} g^{p m} g^{q n} g_{i j} \mp g_{i j}\left[F_{0}^{j}+F_{l}^{j} g^{l m} V_{m}\left(1+g_{r s} V^{r} V^{s}\right)^{-1 / 2}\right]$.

Using this we obtain

$$
\begin{aligned}
\frac{d}{d \tau}\left(g^{i j} V_{i} V_{j}\right) & =2 k^{i j} V_{i} V_{j} \mp 2 g^{i j} V_{i} F_{0 j} \\
& \leq\left(-2 \gamma+C e^{-\gamma \tau}\right) g^{i j} V_{i} V_{j} \mp 2 g^{i j} V_{i} F_{0 j}
\end{aligned}
$$

We have used (3.22) in [4]. From the latter inequality on $\gamma:=(\Lambda / 3)^{1 / 2}$ and $C$ is an arbitrary positive constant which may change from line to line. The second term in the right hand side of (3.48) can be estimated using the Cauchy-Schwarz inequality and the elementary inequality $x y \leq \varepsilon x^{2} / 2+y^{2} / 2 \varepsilon$ :

$$
\begin{aligned}
g^{i j} V_{i} F_{0 j} & \leq\left(g^{i j} V_{i} V_{j}\right)^{1 / 2}\left(g^{i j} F_{0 i} F_{0 j}\right)^{1 / 2} \\
& \leq \frac{\varepsilon}{2}\left(g^{i j} V_{i} V_{j}\right)+\frac{1}{2 \varepsilon}\left(g^{i j} F_{0 i} F_{0 j}\right),
\end{aligned}
$$

where $\varepsilon$ is such that $0<\varepsilon<2 \gamma$. On the other hand from the definition of $\tau_{00}$ it follows that

$$
g^{i j} F_{0 i} F_{0 j} \leq \tau_{00} .
$$

and using (1.37) $g^{i j} F_{0 i} F_{0 j} \leq C e^{-2 \gamma \tau}$. Thus we deduce from (3.48) and (3.49) the following

$$
\frac{d}{d \tau}\left(g^{i j} V_{i} V_{j}\right) \leq\left(-2 \gamma+\varepsilon+C e^{-\gamma \tau}\right) g^{i j} V_{i} V_{j}+C e^{-\gamma \tau} .
$$

Setting $V:=e^{(2 \gamma-\varepsilon) \tau} g^{i j} V_{i} V_{j}$, it follows that

$$
\frac{d V}{d \tau} \leq C e^{-\gamma \tau} V+C e^{-\varepsilon \tau}
$$

$V$ is thus bounded by Gronwall inequality and then

$$
g^{i j} V_{i} V_{j} \leq C e^{(-2 \gamma+\varepsilon) \tau} .
$$

Therefore $g^{i j} V_{i} V_{j}$ is bounded. This is enough to deduce from (3.47) that for $m \geq 0$, we have

$$
\frac{d \nu}{d \tau} \geq C,
$$

so that $\nu$ goes to infinity as does $\tau$. The completeness of causal trajectories is then proved. 


\subsection{Proof of Theorem 1.6}

The proof is based on a bootstrap argument.

By hypothesis

$$
\begin{gathered}
\left|t_{0} \dot{\lambda}\left(t_{0}\right)-1\right| \leq \delta,\left|\left(e^{-\lambda} \mu^{\prime}\right)\left(t_{0}\right)\right| \leq \delta,\left|\left(e^{\lambda} E\right)\left(t_{0}\right)\right| \leq \delta \\
\left|\Lambda t_{0}^{2} e^{2 \mu\left(t_{0}\right)}-3-3 k e^{2 \mu\left(t_{0}\right)}\right| \leq \delta, \bar{w}\left(t_{0}\right) \leq \delta
\end{gathered}
$$

and by continuity, this implies that

$$
\begin{gathered}
|t \dot{\lambda}(t)-1| \leq 2 \delta,\left|\left(e^{-\lambda} \mu^{\prime}\right)(t)\right| \leq 2 \delta,\left|\left(e^{\lambda} E\right)(t)\right| \leq 2 \delta \\
\left|\Lambda t^{2} e^{2 \mu(t)}-3-3 k e^{2 \mu(t)}\right| \leq 2 \delta, \quad \bar{w}(t) \leq 2 \delta
\end{gathered}
$$

with $t$ close to $t_{0}$.

Let $C_{1}$ and $\epsilon$ be constants with $0<C_{1}<1$ and $0<\epsilon<2 / 3$. We can reduce $\delta$ if necessary so that $2 \delta<C_{1} t_{0}^{-3+\epsilon}$. Then there exists some time interval on which the following bootstrap assumption is satisfied

$$
\begin{gathered}
|t \dot{\lambda}-1| \leq C_{1} t^{-2+\epsilon},\left|e^{-\lambda} \mu^{\prime}\right| \leq C_{1} t^{-2+\epsilon},\left|e^{\lambda} E\right| \leq C_{1} t^{-2+\epsilon} \\
\left|\Lambda t^{2} e^{2 \mu}-3-3 k e^{2 \mu}\right| \leq C_{1} t^{-3+\epsilon}, \bar{w}(t) \leq C_{1} t^{-1+\epsilon} .
\end{gathered}
$$

Consider the maximal interval $\left[t_{0}, t_{*}\right)$ on which 3.52 -3.53 hold and suppose $t_{*}$ is finite.

Let us continue with the following set of equations:

$$
\begin{array}{r}
e^{-2 \mu}(2 t \dot{\lambda}+1)+k-\Lambda t^{2}=8 \pi t^{2} \rho \\
e^{-\lambda} \mu^{\prime}=-4 \pi t e^{\mu} j \\
\partial_{t}\left(t^{2} e^{\lambda} E\right)=-t^{2} e^{\mu} b \\
e^{-2 \mu}(2 t \dot{\mu}-1)-k+\Lambda t^{2}=8 \pi t^{2} p \\
\dot{w}=-\dot{\lambda} w-e^{\mu-\lambda} \mu^{\prime} \sqrt{1+w^{2}+L / t^{2}} \mp e^{\lambda+\mu} E .
\end{array}
$$

From (3.54) we have

$$
t \dot{\lambda}-1=\frac{1}{2}\left(\Lambda e^{2 \mu} t^{2}-3-3 k e^{2 \mu}\right)+k e^{2 \mu}+4 \pi t^{2} e^{2 \mu} \rho
$$

from (3.57)

$$
\partial_{t}\left[-\frac{1}{3} t e^{-2 \mu}\left(\Lambda t^{2} e^{2 \mu}-3-3 k e^{2 \mu}\right)\right]=-8 \pi t^{2} p
$$

and from (3.58)

$$
\partial_{t}(t w)=-t^{-1}(t \dot{\lambda}-1)(t w)-t e^{\mu}\left(e^{-\lambda} \mu^{\prime}\right) \sqrt{1+w^{2}+L / t^{2}} \mp t e^{\mu}\left(e^{\lambda} E\right) .
$$


Consider a solution of these equations on the interval $\left[t_{0}, t_{*}\right)$ which satisfies the bootstrap assumption (3.52)-(3.53). Putting inequalities (3.52)-(3.53) into equations (3.55)-(3.56), (3.59)-(3.61) allows new estimates to be derived. For this purpose it is important to have estimates for the matter quantities $p, j$ and $b$.

Let $F:=\max \left\{\left\|f^{+}\right\|_{L^{\infty}},\left\|f^{-}\right\|_{L^{\infty}}\right\}$.

$$
\begin{gathered}
p \leq \frac{\pi}{t^{2}} \int_{-\infty}^{\infty} \int_{0}^{\infty} \frac{w^{2}}{\sqrt{1+w^{2}+L / t^{2}}}\left(f^{+}+f^{-}\right) d L d w \\
\leq 4 \pi \bar{L} F C_{1}^{3} t^{-5+3 \epsilon} \\
|j|=\left|\frac{\pi}{t^{2}} \int_{-\infty}^{\infty} \int_{0}^{\infty} w\left(f^{+}+f^{-}\right) d L d w\right| \\
\leq \frac{4 \pi \bar{L} F}{t^{2}} \bar{w}^{2} \\
\leq 4 \pi \bar{L} F C_{1}^{2} t^{-4+2 \epsilon}, \\
|b|=\left|\frac{\pi}{t^{2}} \int_{-\infty}^{\infty} \int_{0}^{\infty} \frac{w}{\sqrt{1+w^{2}+L / t^{2}}}\left(f^{+}-f^{-}\right) d L d w\right| \\
\leq 4 \pi \bar{L} F C_{1}^{2} t^{-4+2 \epsilon} .
\end{gathered}
$$

An estimate for $e^{2 \mu}$ is also required.

$$
\begin{aligned}
e^{2 \mu} & =\Lambda^{-1} t^{-2}\left(\Lambda t^{2} e^{2 \mu}\right) \\
& \leq \Lambda^{-1} t^{-2}\left[\left(\Lambda t^{2} e^{2 \mu}-3-3 k e^{2 \mu}\right)+3+3 k e^{2 \mu}\right] \\
& \leq \Lambda^{-1} t^{-2}\left[C_{1} t^{-3+\epsilon}+3+3 k e^{2 \mu}\right] .
\end{aligned}
$$

If $k \leq 0$ the last term in the latter inequality can be discarded. If $k>0$ then we need $3 \Lambda^{-1} t^{-2}<1$ i.e. $\Lambda t^{2}>3$. Assume for the moment that $k \leq 0$. Then

$$
\begin{aligned}
e^{2 \mu} & \leq \Lambda^{-1} t^{-2}\left[C_{1} t^{-3+\epsilon}+3\right] \\
& \leq 3 \Lambda^{-1} t^{-2}+C_{1} \Lambda^{-1} t^{-5+\epsilon} .
\end{aligned}
$$

It follows that

$$
\begin{aligned}
\left|4 \pi t e^{\mu} j\right| & \leq 4 \pi t\left[3 \Lambda^{-1} t^{-2}+C_{1} \Lambda^{-1} t^{-5+\epsilon}\right]^{1 / 2} \times 4 \pi \bar{L} F C_{1}^{2} t^{-4+2 \epsilon} \\
& \leq 16 \pi^{2} \bar{L} F C_{1}^{2} t^{-3+2 \epsilon}\left[\sqrt{3 \Lambda^{-1}} t^{-1}+C_{1}^{1 / 2} \Lambda^{-1 / 2} t^{-5 / 2+\epsilon / 2}\right],
\end{aligned}
$$

keeping the worst powers and using (3.55) implies

$$
\left|e^{-\lambda} \mu^{\prime}\right| \leq 16 \pi^{2} \bar{L} F C_{1}^{2}\left(\sqrt{3 \Lambda^{-1}}+C_{1}^{1 / 2} \Lambda^{-1 / 2}\right) t^{-4+2 \epsilon}=: C_{2} t^{-4+2 \epsilon} .
$$


Note that here there is no dependence on the initial data except for the conserved quantities $\bar{L}$ and $F$. Moreover $C_{2}=O\left(C_{1}^{2}\right)$.

From (3.64)-3.65) we have

$$
\begin{aligned}
\left|\partial_{t}\left(t^{2} e^{\lambda} E\right)\right| & =\left|t^{2} e^{\mu} b\right| \\
& \leq\left[3 \Lambda^{-1} t^{-2}+C_{1} \Lambda^{-1} t^{-5+\epsilon}\right]^{1 / 2} \times 4 \pi \bar{L} F C_{1}^{2} t^{-2+2 \epsilon},
\end{aligned}
$$

keeping the worst powers gives

$$
\left|\partial_{t}\left(t^{2} e^{\lambda} E\right)\right| \leq\left(\sqrt{\frac{3}{\Lambda}}+C_{1}^{1 / 2} \Lambda^{-1 / 2}\right) \times 4 \pi \bar{L} F C_{1}^{2} t^{-3+2 \epsilon},
$$

and integrating this in time yields

$$
\begin{aligned}
\left(e^{\lambda} E\right)(t) & \leq t_{0}^{2} e^{\lambda\left(t_{0}\right)}\left|E\left(t_{0}\right)\right| t^{-2}+\frac{1}{2-2 \epsilon}\left(\sqrt{\frac{3}{\Lambda}}+C_{1}^{1 / 2} \Lambda^{-1 / 2}\right) \times 4 \pi \bar{L} F C_{1}^{2} t^{-4+2 \epsilon} \\
& \leq\left[t_{0}^{2} e^{\lambda\left(t_{0}\right)}\left|E\left(t_{0}\right)\right|+\frac{2 \pi}{1-\epsilon}\left(\sqrt{\frac{3}{\Lambda}}+C_{1}^{1 / 2} \Lambda^{-1 / 2}\right) \bar{L} F C_{1}^{2}\right] t^{-2}=: C_{3} t^{-2} .
\end{aligned}
$$

The constant $C_{3}$ in the last inequality depends in a transparent way on the initial data. We have

$$
8 \pi t^{2} p \leq 32 \pi^{2} \bar{L} F C_{1}^{3} t^{-3+3 \epsilon},
$$

so that using (3.60) and integration gives

$$
\begin{array}{r}
\left|-\frac{1}{3} t e^{-2 \mu}\left(\Lambda t^{2} e^{2 \mu}-3-3 k e^{2 \mu}\right)\right| \leq \frac{1}{3} t_{0} e^{-2 \mu\left(t_{0}\right)}\left|\Lambda t_{0}^{2} e^{2 \mu\left(t_{0}\right)}-3-3 k e^{2 \mu\left(t_{0}\right)}\right| \\
+\left|\frac{32 \pi^{2}}{-2+3 \epsilon} \bar{L} F C_{1}^{3} t^{-2+3 \epsilon}\right| .
\end{array}
$$

At this point the assumption $\epsilon<2 / 3$ is needed. Then

$$
\left|-\frac{1}{3} t e^{-2 \mu}\left(\Lambda t^{2} e^{2 \mu}-3-3 k e^{2 \mu}\right)\right| \leq \frac{1}{3} t_{0}\left|\Lambda t_{0}^{2}-3 e^{-2 \mu\left(t_{0}\right)}-3 k\right|+\mid \frac{32 \pi^{2}}{2-3 \epsilon} \bar{L} F C_{1}^{3} .
$$

Using (3.65) and keeping the worst powers, it follows that

$$
\begin{aligned}
\left|\Lambda t^{2} e^{2 \mu}-3-3 k e^{2 \mu}\right| & \leq\left[3 \Lambda^{-1}+C_{1} \Lambda^{-1}\right]\left[t_{0}\left|\Lambda t_{0}^{2}-3 e^{-2 \mu\left(t_{0}\right)}-3 k\right|+\frac{96 \pi^{2}}{2-3 \epsilon} \bar{L} F C_{1}^{3}\right] t^{-3} \\
& =: C_{4} t^{-3}
\end{aligned}
$$

Now let us examine the evolution of $w$.

Using (3.65), (3.66), (3.67), the bootstrap assumption, the fact that $C_{1}<1$ and 
then keeping the worst powers gives

$$
\begin{aligned}
\mid-t e^{\mu}\left(e^{-\lambda} \mu^{\prime}\right) & \sqrt{1+w^{2}+L / t^{2}} \pm t e^{\mu}\left(e^{\lambda} E\right) \mid \\
\leq & {\left[3 t_{0}^{2} e^{\lambda\left(t_{0}\right)}\left|E\left(t_{0}\right)\right|+18 \pi \bar{L} F \Lambda^{-1 / 2} C_{1}^{2}\left(\frac{1}{1-\epsilon}+8 \pi \Lambda^{-1 / 2}(2+\sqrt{\bar{L}})\right)\right] t^{-2}, }
\end{aligned}
$$

and using (3.61), the bootstrap assumption and integration it follows that

$$
\begin{aligned}
t \bar{w}(t) & \leq t_{0} \bar{w}\left(t_{0}\right)+\int_{t_{0}}^{t} C_{1} s^{-3+\epsilon} s \bar{w}(s) d s \\
& +\int_{t_{0}}^{t}\left[3 t_{0}^{2} e^{\lambda\left(t_{0}\right)}\left|E\left(t_{0}\right)\right|+18 \pi \bar{L} F \Lambda^{-1 / 2} C_{1}^{2}\left(\frac{1}{1-\epsilon}+8 \pi \Lambda^{-1 / 2}(2+\sqrt{\bar{L}})\right)\right] s^{-2} d s \\
& \leq t_{0} \bar{w}\left(t_{0}\right)+6 t_{0}^{2} e^{\lambda\left(t_{0}\right)}\left|E\left(t_{0}\right)\right|+36 \pi \bar{L} F \Lambda^{-1 / 2} C_{1}^{2}\left(\frac{1}{1-\epsilon}+8 \pi \Lambda^{-1 / 2}(2+\sqrt{\bar{L}})\right) \\
& +\frac{C_{1}^{2}}{1-\epsilon}=: C_{5} .
\end{aligned}
$$

An estimate on the matter quantity $\rho$ is needed. Using (3.69) gives

$$
\begin{aligned}
& \rho_{v l}:=\frac{\pi}{t^{2}} \int_{-\infty}^{\infty} \int_{0}^{\infty} \sqrt{1+w^{2}+L / t^{2}}\left(f^{+}+f^{-}\right) d L d w \\
& \leq \frac{\pi}{t^{2}} \int_{-\bar{L}}^{\bar{L}} \int_{0}^{\bar{w}} \sqrt{1+\bar{w}^{2}+L / t^{2}} 2 F d L d w \\
& \leq \frac{4 \pi \bar{L} F}{t^{2}} \bar{w} \sqrt{1+\bar{w}^{2}+L / t^{2}} \\
& \leq \frac{4 \pi \bar{L} F}{t^{2}} C_{5} t^{-1} \sqrt{1+C_{5}^{2} t^{-2}+\bar{L} t^{-2}} \\
& \leq 4 \pi \bar{L} F C_{5} t^{-3}\left(1+C_{5} t^{-1}+\sqrt{\bar{L}} t^{-1}\right), \\
& \rho:=\rho_{v l}+ \frac{1}{2}\left(e^{\lambda} E\right)^{2}+\frac{1}{2} c t^{-4} \\
& \leq 4 \pi \bar{L} F C_{5} t^{-3}\left(1+C_{5} t^{-1}+\sqrt{\bar{L}} t^{-1}\right)+\frac{1}{2} C_{1}^{2} t^{-4+2 \epsilon}+\frac{1}{2} c t^{-4} .
\end{aligned}
$$

In the case of plane symmetry $k=0$ equations (3.65), (3.68) and (3.71) imply that

$$
\begin{aligned}
& \left|\frac{1}{2}\left(\Lambda e^{2 \mu} t^{2}-3-3 k e^{2 \mu}\right)+k e^{2 \mu}+4 \pi t^{2} e^{2 \mu} \rho\right| \\
& \quad \leq \frac{1}{2} C_{4} t^{-3}+4 \pi t^{2}\left[3 \Lambda^{-1} t^{-2}+C_{1} \Lambda^{-1} t^{-5+\epsilon}\right] \times \\
& \quad\left[4 \pi \bar{L} F C_{5} t^{-3}\left(1+C_{5} t^{-1}+\sqrt{\bar{L}} t^{-1}\right)+\frac{1}{2} C_{1}^{2} t^{-4+2 \epsilon}+\frac{1}{2} c t^{-4}\right] \\
& \quad \leq\left[\frac{1}{2} C_{4}+16 \pi^{2} \bar{L} F \Lambda^{-1} C_{5}\left(3+C_{1}\right)\left(1+C_{5}+\sqrt{\bar{L}}\right)+\frac{1}{2} C_{1}^{2}+\frac{1}{2} c\right] t^{-2} .
\end{aligned}
$$


For the latter inequality we only kept the worst powers. We have

$$
|t \dot{\lambda}-1| \leq C_{6} t^{-2},
$$

where $C_{6}$ is the constant written out in (3.72) above.

The constants $C_{2}-C_{6}$ appearing in equations (3.69)-(3.73) are all less than or equal to $C \times\left(g(\delta)+C_{1}^{2}\right)$, with $C$ a positive constant and $g(\delta)$ a positive function of $\delta$ tending to 0 as $\delta$ tends to 0 . Therefore it is always possible to choose $C_{1}$ and $\delta$ small enough in such a way that $C C_{1} \leq 1 / 2$ and $C g(\delta) \leq C_{1} / 2$, and so the constants $C_{2}-C_{6}$ are all less than $C_{1}$. This closes the bootstrap argument as it implies that (3.52)-(3.53) hold on an interval $\left[t_{0}, t_{1}\right)$, with $t_{1}>t_{*}$. This contradicts the maximality of the interval $\left[t_{0}, t_{*}\right)$. Therefore $t_{*}=\infty$. To complete the proof of theorem 1.6] it remains to show that the spacetime is complete. In fact recall that as in 12 the relation between coordinate time $t$ and proper time $\tau$ along the trajectory is given by

$$
\frac{d \tau}{d t}=\frac{e^{\mu}}{\sqrt{m^{2}+w^{2}+L / t^{2}}} .
$$

The decay estimate on $e^{\lambda} E$ can be used to obtain the inequalities

$$
e^{2 \mu} \geq \frac{t}{C+(C-k) t+\frac{\Lambda}{3} t^{3}}, \text { for } k \leq 0,
$$

and

$$
e^{2 \mu} \geq \frac{t}{C+C t+\frac{\Lambda}{3} t^{3}}, \text { for } k=1
$$

The following estimate follows

$$
e^{\mu} \geq C t^{-1}, t \geq t_{0}
$$

Thus

$$
\frac{d \tau}{d t} \geq \frac{C t^{-1}}{\sqrt{m^{2}+C+L}},
$$

and so $\tau$ goes to infinity as does $t$. Theorem [1.6 is then proved.

Remark In the case of hyperbolic symmetry $k=-1$, the same argument works provided $\Lambda$ is sufficiently large. Indeed in that case $\Lambda^{-1}\left(3+C_{1}\right) t^{-2}$ appears as an adding term in the right hand side of equation (3.72).

Acknowledgement : The author thanks A.D. Rendall for fruitful suggestions.

\section{References}

[1] H. Andréasson, The Einstein-Vlasov system/kinetic theory, Liv. Rev. Relativity 8, lrr-2005-2 (2005) 
[2] H. Andréasson, G. Rein and A.D. Rendall, On the Einstein-Vlasov system with hyperbolic symmetry, Math. Proc. Camb. Phil. Soc. 134, 529-549 (2003)

[3] H. Andréasson, A.D. Rendall and M. Weaver, Existence of CMC and constant areal time foliations in $T^{2}$ symmetric spacetimes with Vlasov matter, Communications in Partial Differential Equations 29, 237-262 (2004)

[4] H. Lee, Asymptotic behaviour of the Einstein-Vlasov system with a positive cosmological constant, Math. Proc. Camb. Phil. Soc. 137, 495-509 (2004)

[5] P. Noundjeu, The Einstein-Vlasov-Maxwell system with spherical symmetry, Class. Quantum Grav. 22, 5365-5384 (2005)

[6] G. Rein, The Vlasov-Einstein system with surface symmetry, Habilitationsschrift Ludwig-Maximilians-Universität München 1995

[7] G. Rein, Cosmological solutions of the Vlasov-Einstein system with spherical, plane, and hyperbolic symmetry, Math. Proc. Camb. Phil. Soc. 119, 739-762 (1996)

[8] A.D. Rendall, Crushing singularities in spacetimes with spherical, plane and hyperbolic symmetry, Class. Quantum Grav. 12, 1517-1533 (1995)

[9] A.D. Rendall and C. Uggla, Dynamics of spatially homogeneous locally rotationally symmetric solutions of the Einstein-Vlasov equations, Class. Quantum Grav. 17, 4697-4713 (2000)

[10] A.D. Rendall, An introduction to the Einstein-Vlasov system, Banach Center Publications 41, 35-68 (1997)

[11] H. Ringström, On the $T^{3}$-Gowdy symmetric Einstein-Maxwell equations, Ann. Henri Poincaré 7, 1-20 (2006)

[12] S.B. Tchapnda and A.D. Rendall, Global existence and asymptotic behaviour in the future for the Einstein-Vlasov system with positive cosmological constant, Class. Quantum Grav. 20, 3037-3049 (2003)

[13] S.B. Tchapnda and N. Noutchegueme, The surface-symmetric Einstein-Vlasov system with cosmological constant, Math. Proc. Camb. Phil. Soc. 138, 541$553(2005)$

[14] R.M. Wald, Asymptotic behavior of homogeneous cosmological models in the presence of a positive cosmological constant, Phys. Rev. D 28, 2118-2120 (1983)

[15] T. Zhou, Y. Guo and C.-W. Shu, Numerical study on Landau damping, Physica D 157, 322-333 (2001) 
Sophonie Blaise Tchapnda

Max Planck Institute for Gravitational Physics

Albert Einstein Institute

Am Mühlenberg 1, D-14476 Golm, Germany

email: tchapnda@aei.mpg.de

On leave from: Department of Mathematics, Faculty of Science

University of Yaounde I, PO Box 812, Yaounde, Cameroon

email: tchapnda@uycdc.uninet. $\mathrm{cm}$ 